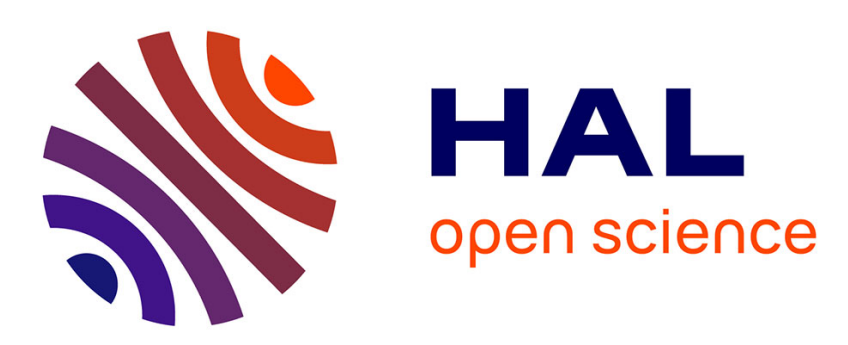

\title{
Docking de protéines en réalité virtuelle. Une approche hybride et multimodale
}

Nicolas Férey, Guillaume Bouyer, Christine Martin, Abdelhamid Drif, Patrick Bourdot, Mehdi Ammi, Julien Nelson, Jean-Marie Burkhardt, Ludovic Autin

\section{- To cite this version:}

Nicolas Férey, Guillaume Bouyer, Christine Martin, Abdelhamid Drif, Patrick Bourdot, et al.. Docking de protéines en réalité virtuelle. Une approche hybride et multimodale. Revue des Sciences et Technologies de l'Information - Série TSI : Technique et Science Informatiques, 2009, 28 (8), pp.983 1015. 10.3166/tsi.28.983-1015 . hal-01753902

\section{HAL Id: hal-01753902 \\ https://hal.science/hal-01753902}

Submitted on 29 Mar 2018

HAL is a multi-disciplinary open access archive for the deposit and dissemination of scientific research documents, whether they are published or not. The documents may come from teaching and research institutions in France or abroad, or from public or private research centers.
L'archive ouverte pluridisciplinaire HAL, est destinée au dépôt et à la diffusion de documents scientifiques de niveau recherche, publiés ou non, émanant des établissements d'enseignement et de recherche français ou étrangers, des laboratoires publics ou privés. 


\title{
Docking de protéines en Réalité Virtuelle : une approche hybride et multimodale
}

\author{
N. Férey * - G. Bouyer * - C. Martin * - A. Drif * - P. Bourdot * \\ - M. Ammi ${ }^{*}-$ J. Nelson ${ }^{\dagger}-$ J-M. Burkhart ${ }^{\dagger}-$ L. Autin $\ddagger$ \\ * IBISC-CNRS, FRE 3190, Université d'Evry-Val d'Essonne, Génopole \\ nicolas.ferey@ibpc.fr \\ * LIMSI-CNRS, UPR 3251, Université Paris-Sud XI \\ \{bouyer,martinc,drif,bourdot,ammi\}@limsi.fr \\ $\dagger$ ECI, EA 4070, Université Paris V \\ \{jean-marie.burkhardt, julien.nelson\}@univ-paris5.fr \\ $\ddagger$ EBGM, INSERM UMRS 726 \\ ludovic.autin@gmail.com
}

RÉSUMÉ. Le docking de protéines in-silico est la détermination de la structure $3 d$ des complexes protéiques à l'échelle atomique, qui permet de mieux comprendre la fonction biologique de ces complexes. Dans le cadre du projet ANR CoRSAIRe, notre hypothèse est que les interactions multimodales en Réalité Virtuelle $(R V)$ sont susceptibles d'améliorer la rapidité de ces dockings. A cet effet, nous avons conduit une analyse ergonomique afin d'identifier les besoins des biologistes sur les systèmes de docking actuels. Puis nous avons conçu un modèle et une application immersive et multimodale dans laquelle les rendus visuel, audio et haptique sont combinés pour transmettre les informations nécessaires à ce type d'activité.

ABSTRACT. Protein docking studies how proteins combine with each other in $3 d$, in order to better understand their biological functions. The basic hypothesis of the french research projet CoRSAIRe is that Virtual Reality (VR) multimodal interactions can increase efficiency in reaching docking solutions. To this end, we have conducted an ergonomic analysis of the protein-protein current docking task. Using these results, we have designed an immersive and multimodal application where visual, audio and haptic feedbacks are combined to communicate biological information, help manipulating proteins and exploring possible solutions of assembly.

MOTS-CLÉS : Docking, Conception Centrée Utilisateur, Réalité Virtuelle, Rendus Multimodaux KEYWORDS: Docking, User-Centered Design, Virtual Reality, Multimodal Rendering

2e soumission à $R S T I$, le 03/09/08 


\section{Introduction}

Le docking ou amarrage protéine-protéind 1 est un problème de biologie structurale qui, à partir des structures tridimensionnels (3d) des protéines et de leurs propriétés physico-chimiques (section 22, consiste à prédire comment les protéines s'associent entre elles pour former des complexes fonctionnels au niveau de la cellule. La connaissance de la structure des complexes protéine-protéine permet aux chercheurs de mieux comprendre les mécanismes clefs mis en jeux lors d'une interaction entre protéines, ce qui constitue un enjeu scientifique majeur, autant en termes fondamentaux (expliquer une fonction cellulaire) que pour la recherche appliquée (inhiber spécifiquement une fonction cellulaire).

Actuellement, les méthodes employées pour étudier le docking combinent (1) des phases automatiques (explicitées en section 3.1) prenant en compte les paramètres topologiques ainsi qu'énergétiques (physico-chimiques) des protéines, et (2) des phases de visualisation moléculaire permettant d'évaluer les résultats obtenus. La phase automatique est coûteuse en temps de calcul, et fournit un grand nombre de solutions de docking potentielles difficilement discriminables juste sur la base des paramètres évalués. C'est pourquoi une phase d'analyse visuelle manuelle par un expert est nécessaire afin d'obtenir un résultat pertinent. Néanmoins cette analyse est très lourde en terme de quantité d'information qu'il faut prendre en compte simultanément (manipulation d'objet $3 \mathrm{~d}$, caractéristiques physico-chimiques, données biologiques, etc.).

Du fait des limitations actuelles des outils servant au docking, il est important de développer des approches complémentaires et/ou alternatives. Dans le cadre du projet ANR CoRSAIRe (Combinaison des Rendus Sensori-moteurs pour l'Analyse Immersive de Résultats), notre hypothèse est que l'utilisation des technologies et des interactions de Réalité Virtuelle (RV) peut aider les chercheurs dans leurs activités de docking. En premier lieu, la stéréoscopie, surtout lorsqu'elle est adaptative, améliore la perception des modèles $3 \mathrm{~d}$ des protéines. De plus, la manipulation directe de plusieurs protéines en même temps dans l'espace, offerte par les dispositifs couramment utilisés dans ce contexte (souris $3 \mathrm{~d}$, bras à retour d'effort, etc.) est plus intuitive et plus efficace que les interfaces de bureau classiques (souris, clavier). Par ailleurs, la multimodalité en sortie (approche visant à spécifier l'adaptation dynamique des rendus visuels, haptiques et audio aux caractéristiques des informations) est une réponse possible au problème de la quantité importante de paramètres à présenter simultanément à l'utilisateur durant le docking et l'analyse de ses résultats. Enfin, l'approche fortement interactive du docking en RV est une occasion de recentrer l'utilisateur expert du domaine dans l'étude de ce phénomène, et non plus de confier entièrement la génération des solutions possibles à un algorithme automatique. Nous pensons que cette approche, combinant les bénéfices des interactions multimodales, de la compétence des chercheurs experts (biologiste, cristallographe, bioinformaticien, modélisateur),

1. Dans la suite de cet article nous emploierons l'anglicisme "docking", largement accepté dans la communauté des biologistes francophones. 
permettra d'améliorer la rapidité de prédiction des complexes protéine-protéine ainsi que l'efficacité et la qualité de leurs analyses.

Des applications de docking interactif, multimodal ou immersif, ont déjà été développées et sont présentées dans la section 3.2. Néanmoins, ces travaux semblent pénalisés par un manque d'informations préalables claires sur les habitudes et les besoins des utilisateurs. Pour cela, nous avons conduit des analyses ergonomiques de la tâche de docking de protéines telle qu'elle est réalisée actuellement par les experts, afin de déterminer les avantages et les inconvénients des outils existants, et d'aider à la conception de paradigmes d'interaction multimodale pertinents. Les données, les recommandations et les scénarii de travail issus de ces analyses (section 4) nous ont permis de modéliser une approche hybride complète pour le docking mutualisant phases d'interactions et phases automatiques. Cette approche a ensuite été mise en application dans un premier prototype, dont nous présentons l'architecture, les contraintes et les solutions techniques retenues (section 5.2 . Nous sommes actuellement dans la phase d'évaluation de ce prototype en collaboration avec des chercheurs experts en docking (section 6).

\section{Problématique du docking de protéines}

\subsection{Caractéristiques des protéines}

Les protéines jouent le rôle à la fois de briques de base et d'ouvriers de la cellule. Elles sont synthétisées à partir d'une portion d'ADN (Acide Désoxyribo-Nucléique) appelée séquence codante ou gène qui est transcrite en ARN messager (Acide RiboNucléique messager). Cette ARN messager (ARNm) est alors traduit en protéine, grâçe au ribosome, via le code génétique (figure 1A). A chaque triplet de paire de base d'ARNm va correspondre un acide aminé (ou résidu). En effet, il existe 20 acides aminés qui se différencient par la nature chimique du groupement latéral $(R)$ ou chaine latérale. Il est ainsi possible de les classer en différents groupes partageant certaines caractéristiques, qui vont induire des comportements différents (figure 1A) : chargés, apolaires (ou hydrophobes), polaires (ou hydrophiles).... Ce sont ces différentes propriétés physico-chimiques qui vont donner lieu aux interactions atomiques induisant le repliement de la protéine et sa stabilité (figure 1B). Notons, que ces propriétés jouent également un rôle très important lors des interactions entre protéines.

Les protéines sont donc de longues chaînes composées d'une succession d'acides aminés repliés dans l'espace et constituent ainsi l'expression du programme génétique d'un organisme. Mais, afin d'exercer leur fonction au sein de la cellule, les protéines synthétisées doivent se replier dans l'espace et adopter une forme $3 \mathrm{~d}$ spécifique. Cette forme se caractérise par quatre niveaux de structure (figure 1B). L'ordre d'enchaînement ou séquence des résidus constitue la structure primaire (figure $1 \mathrm{~B}-\mathrm{a}$ ). Certains segments de structure s'organisent en une séquence de sous-structures spécifiques appelées les structures secondaires (figure 1B-b). Ces structures, chimiquement plus stables grâce aux liaisons hydrogène, se décomposent en deux groupes : (1) les struc- 


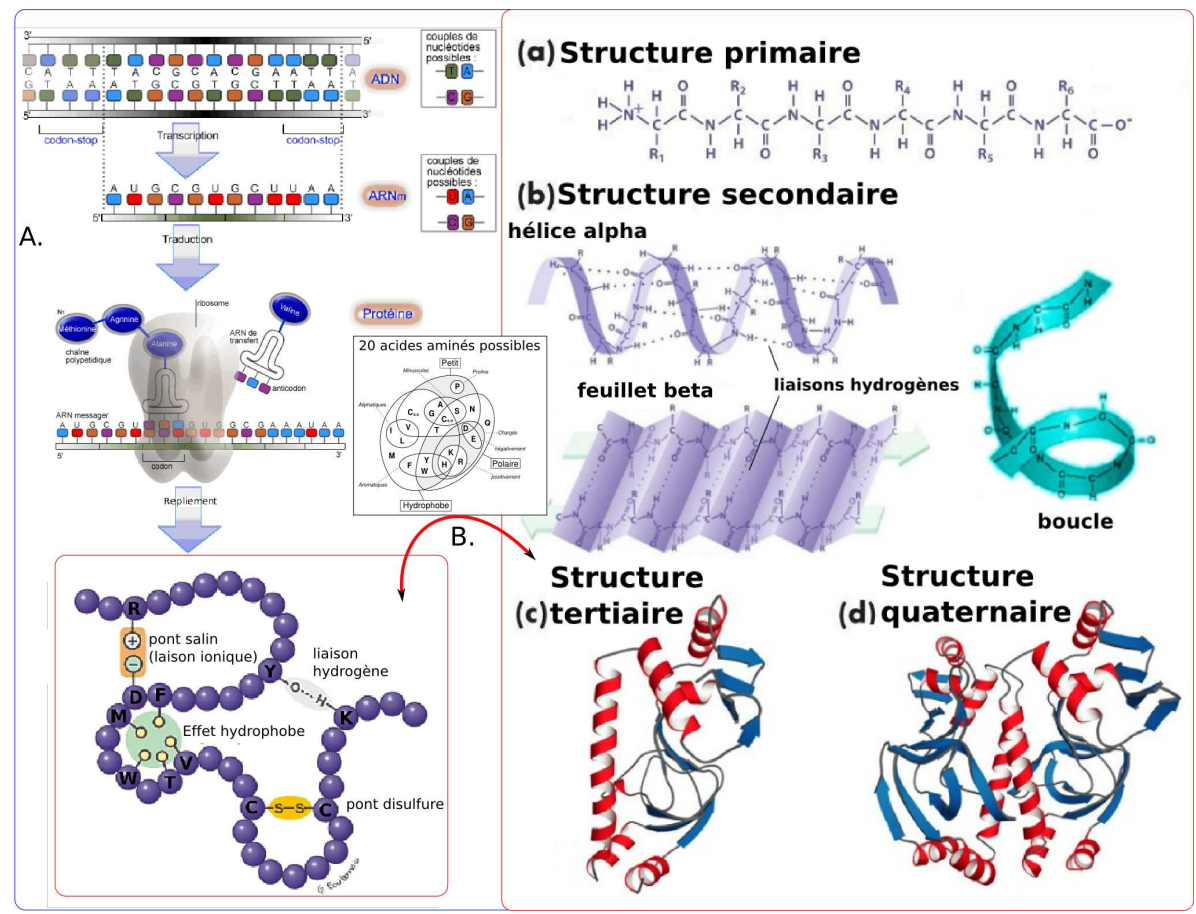

Figure 1. (A) - Schéma général de la synthèse des protéines : transcription de l'ADN en ARN messager (ARNm), puis traduction de l'ARNm en séquence d'acides aminés choisis parmi les 20 existants, classés ici selon leurs propriétés physicochimiques(diagramme de Venn).(B) - Du fait de la nature chimique des acides aminés et les interactions en résultant, la protéine se replie dans l'espace. Cette forme $3 d$ repliée se divise en quatre niveaux de structure : (a) primaire, (b) secondaire, (c) tertiaire ou $3 d$ et enfin (d) quaternaire.

tures secondaires régulières hélices alpha $(\alpha)$ et feuillets beta $(\beta)$ qui sont connectées par (2) les structures secondaires irrégulières ou boucles. L'emboîtement de ces structures secondaires va constituer la structure tertiaire ou $3 d$ (figure 1B-c) qui est porteuse de la fonction cellulaire.

Une fois repliées, les protéines remplissent des fonctions très diverses au sein de la cellule, telles que : le transport de molécules entre les différents composants de l'organisme (hémoglobine, chaperonne), la signalisation et la communication inter et intracellulaire (hormone, neurotransmetteur, ions), le processus de défense immunitaire (immunoglobuline, molécule d'adhésion) ou encore le métabolisme cellulaire (chlorophylle, apoptose, facteur de transcription, synthèse d'ATP). Ces fonctions cellulaires sont étroitement liées à la structure tertiaire mais aussi aux interactions avec d'autres protéines. Ces interactions forment de nouvelles entités appelées complexes 
protéiques ou supramoléculaires (exemples figure 2). Ces assemblages constituent la structure quaternaire des protéines (figure 1B-d).

A.

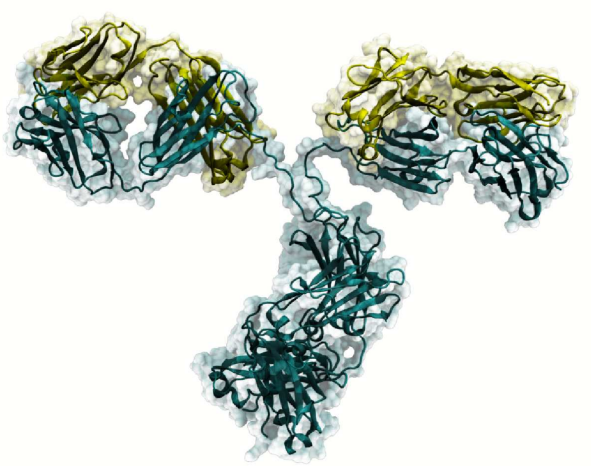

B.

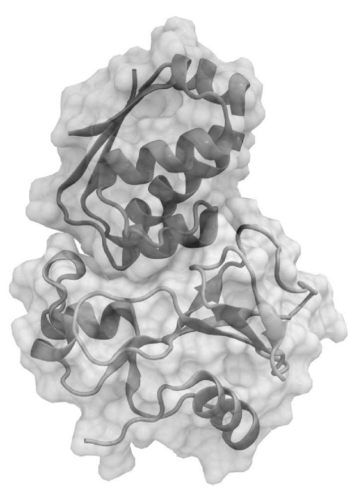

Figure 2. (A) - Structure quaternaire d'un anticorps (immunoglobuline) constituée d'une chaîne lourde et d'une chaîne légère. C'est cet assemblage qui est capable de reconnaître les antigènes des corps étrangers afin de former un complexe supramoléculaire. (B) - Le complexe protéique formé par une enzyme, la Barnase (en bas) et son substrat, la Barstar (en haut). Les structures secondaires et une surface moléculaire transparente du complexe sont représentées afin d'illustrer les complémentarités.

De fait, mieux comprendre les interactions inter-protéines au niveau atomique est un enjeu majeur pour la recherche biomédicale. En effet, les médicaments développés actuellement ciblent de plus en plus des interactions protéine-protéine spécifiques (Villoutreix et al., Apr 2008) ou encore résident dans la synthèse de protéines recombinantes censées reproduire l'interaction de la protéine naturelle (Pipe, May 2008). Il est donc nécessaire de pouvoir résoudre la structure $3 \mathrm{~d}$ des protéines. Il existe à l'heure actuelle deux méthodes expérimentales permettant de déterminer la structure $3 \mathrm{~d}$ d'une protéine ou d'un complexe. Ce sont la cristallographie aux rayons X et la Résonance Magnétique Nucléaire (RMN). Toutes les structures connues sont stockées sur le site de la Protein Data Bank (PDB) (Berman et al., 2000). Cette base comporte environ 50000 structures pour tout type d'organisme. Ce chiffre reste néanmoins très faible au regard de la quantité de protéines existantes dans le monde vivant (estimée par exemple à environ 20000 pour l'Homme). Cela est dû au fait que la détermination expérimentale de la structure d'une protéine est très difficile, parfois impossible. En effet, la résolution d'une structure implique d'arriver à produire la protéine d'intérêt en quantité suffisante, à la purifier et dans le cas de la cristallographie il est nécessaire d'obtenir des cristaux diffractants. De plus, il est impossible d'obtenir des structures utilisables de bonne résolution pour les protéines transmembranaires. Lorsque l'on veut déterminer la structure d'un complexe, les difficultés de production et de purification sont d'autant plus importantes que l'on dispose de plusieurs protéines, qui doivent souvent être produites simultanément pour que le complexe se forme. De plus, le temps nécessaire à la formation d'un cristal est parfois incompatible avec la durée 
de vie de certains complexes. C'est pour ces raisons que de nombreux chercheurs ont cherché à prédire la structure de ces complexes grâce à l'informatique, via les méthodes et algorithmes de docking. Ces méthodes sont fondées sur deux critères issus des connaissances acquises via les complexes existants dans la PDB : critère géométrique/topologique et critère énergétique.

\subsection{Caractéristiques des interfaces protéine-protéine}

\subsubsection{Géométrie et surface}

Un des premiers critères des interfaces protéine-protéine à avoir été étudié est la topologie surfacique du complexe protéique. Il s'avère que la grande majorité des structures $3 \mathrm{~d}$ des complexes protéine-protéine connus présentent une bonne complémentarité topologique ou de surface (ex. figure $2 \mathrm{~B}$ ). Il a également été démontré que l'on pouvait admettre que la valeur de l'aire de surface à l'interface était comprise entre 1000 et $2500 \AA^{2}$. Ce critère a permis de développer les premiers logiciels de docking, uniquement fondés sur une reconnaissance de forme (Connolly, 1983a) (complémentarité de surface moléculaire). Cette approche reste intéressante pour les problèmes de docking de protéines complexes mais rigides. Nous verrons que cette notion peut être utilisée de différentes manières dans un contexte de RV multimodale. Néanmoins, il s'est avéré que le critère géométrique n'était pas suffisant afin de prédire la structure d'un complexe donné. Aussi des méthodes utilisant des critères énergétiques sont intégrées aux logiciels de docking plus récents.

\subsubsection{Physico-chimie : notion d'énergie}

Les complexes protéine-protéine semblent suivrent le principe que la conformation active est la forme pour laquelle l'énergie libre est minimale (Wang et al., 2003). Afin d'évaluer cette énergie libre d'interaction à partir de la structure $3 \mathrm{~d}$, les chercheurs font appel à la mécanique moléculaire. D'un certain point de vue, on peut considérer la mécanique moléculaire comme un système expert constitué d'un modèle physique et d'un ensemble de paramètres permettant de représenter différentes molécules. Cet ensemble est appelé un champ de forces, et décompose les interactions mises en jeu entre les atomes. Pour ce faire, les atomes sont considérés comme des sphères reliées par des ressorts. On distingue les interactions liantes (entre deux atomes connectés par une liaison covalente) des interactions non liantes (entre deux atomes non connectés) qui nous intéressent plus particulièrement puisque ces interactions constituent l'essentiel des interactions protéine-protéine. Ces interactions non-liantes sont le potentiel de Van der Waals et le potentiel électrostatique. A ces potentiels issus d'un champ de force il est possible d'ajouter des composantes représentant d'autres phénomènes importants pour les interactions protéine-protéine. On citera les liaisons hydrogènes ou encore l'effet hydrophobe, ainsi que les caractéristiques géométriques (aire de l'interface et complémentarité). L'énergie libre d'interaction entre deux protéines peut ainsi être approximée par la somme de ces différents potentiels, on parle alors de score. Dans un contexte de docking immersif en temps réel, le choix des équations et des 
méthodes utilisées pour l'évaluation de l'énergie ou du score d'un complexe protéineprotéine est crucial (Wang et al., 2003).

\subsubsection{Interactions de Van der Waals}

Les interactions de Van der Waals sont une approximation empirique des interactions entre atomes. La force de Van der Waals, obtenue par gradient sur le champ de potentiel, est définie par l'équation du potentiel de Lennard-Jones (figure 3). Dans cette équation, $r$ est la distance entre les atomes considérés, $\epsilon$ la distance atome-atome pour laquelle le potentiel devient nul, et $\sigma$ la profondeur du puit de potentiel. $\epsilon$ et $\sigma$ sont déterminés de manière empirique et dépendent de la paire d'atomes considérés. Ce potentiel de Van der Waals contient une composante attractive lorsque les atomes sont liés, et une composante répulsive si les atomes sont trop proches. Il peut être utilisé pour empêcher l'interpénétration de deux protéines durant un docking interactif, en calculant les forces entre les atomes à l'interface (deuxième équation de la figure 3).

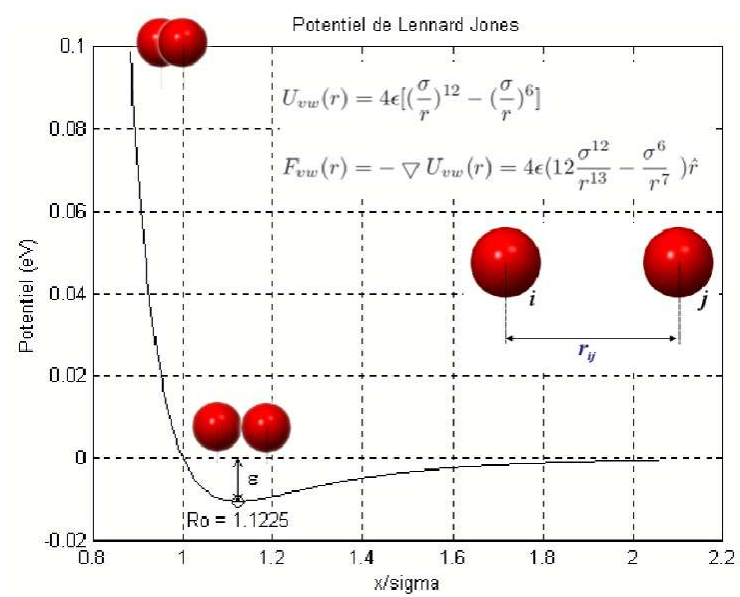

Figure 3. Potentiel, force et profil des interactions de Van der Waals.

\subsubsection{Interactions électrostatiques}

Certains acides aminés peuvent être chargés positivement ou négativement, ce qui donne lieu à des phénomènes électrostatiques qui favorisent la formation d'un complexe protéique spécifique. Ces interactions électrostatiques agissent à "longues" distances (autour de $10 \AA$ A), entre des groupes d'atomes électriquement chargés. L'électrostatique classique dans sa plus simple forme, à savoir les charges dans le vide (ou un milieu homogène), est décrite par l'équation de Poisson (équation 1) qui donne une relation locale entre la distribution de charge et le potentiel :

$$
U_{e l}(r)=-\nabla \bullet \nabla \phi(r)=\frac{\rho(r)}{\epsilon_{0}} \text { et } \vec{E}(r)=-\nabla \phi(r)
$$


$\phi(r)$ et $\rho(r)$ sont respectivement le potentiel électrostatique et la densité de charge en fonction de la position $r$ (coordonnées cartésiennes d'un point dans l'espace), et $\epsilon_{0}$ est la permittivité électrique du vide. Cette équation traduit le fait que les charges sont la source du champ électrostatique $\vec{E}(r)$ (qui est l'opposé du gradient du potentiel).

Si l'on considère l'interaction entre 2 charges ponctuelles dans le vide, la résolution de l'équation de Poisson amène à évaluer l'énergie potentielle $\left(U_{e l}\right)$ par la loi de Coulomb (équation de la figure 4) avec $r$ la distance entre les barycentres des charges $q_{1}$ et $q_{2}$ des atomes considérés, et $\epsilon_{0}$ est la constante de permittivité du vide. Ce potentiel peut être traduit en force $\left(F_{e l}\right)$ utilisable par la modalité haptique (figure 4).

Néanmoins, les protéines ne sont pas dans le vide mais évoluent dans un solvant, l'eau. Ce système protéine-solvant consiste donc en un milieu non-homogène qui comporte une frontière diélectrique, aussi la loi de Coulomb ne convient plus. Lorsqu'une charge est plongée dans l'eau (ou un solvant chargé), elle va attirer les molécules d'eau et ainsi s'en entourer.

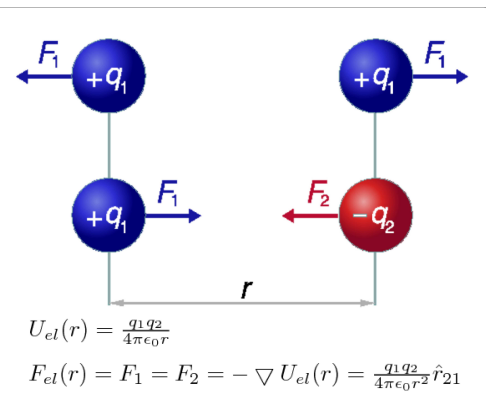

Figure 4. Forces résultantes de l'interaction entre deux charges ponctuelles (de même signe et de signe opposé) selon la loi de Coulomb. Note : $\left(\frac{1}{4 \pi \epsilon_{0}}\right)$ est aussi appelé $K_{c}$ la constante de Coulomb. C'est ce que l'on appelle la solvatation, ou dans le cas de l'eau, l'hydratation. Pour un système complexe, comme une protéine dans l'eau, ou un complexe protéique, il est possible d'utiliser le concept de continuum électrostatique afin d'inclure ce phénomène dans le calcul des interactions électrostatiques. Dans le cadre de notre projet, nous nous sommes intéressés à deux méthodes particulières qui permettent d'évaluer l'électrostatique dans un milieu non-homogène. La méthode la plus simple est une approximation de la loi de Coulomb qui utilise une constante diélectrique dépendante de la distance (en suivant une fonction linéaire avec $K c=\frac{1}{4 \pi \epsilon_{0} \epsilon_{r}}$ et $\left.\epsilon_{r}=f(r)\right)$. Il faut noter que l'on introduit généralement un seuil (cut-off) (par exemple de l'ordre de 8 à $10 \AA ̊$ ) qui a pour conséquence que ces termes sont sans effet au-delà d'une certaine distance entre les atomes.

\subsubsection{Interactions additionelles}

Afin de rendre compte plus finement des interactions protéine-protéine, d'autres critères fondés sur l'énergétique peuvent être pris en compte :

- l'effet hydrophobe : plusieurs acides aminés hydrophobes (formant un "patch" hydrophobe, exemple figure 1B) en contact à l'interface ("complémentarité hydrophobe") entraînent une interaction stabilisante pour beaucoup de complexes.

- liaison hydrogène (exemple figure $1 \mathrm{~B}$ ) : c'est une des interactions qui a le plus fort poids en terme d'énergie. En moyenne, il existe 5 à 6 liaisons hydrogènes par 
interface. On rajoute souvent un terme décrivant cette interaction au champ de force (composante dérivé du potentiel de Lennard-Jones). Mais il est possible de se contenter d'une observation de l'interface.

\section{Docking in silico : état de l'art}

\subsection{Approches automatiques pour le docking de protéine}

Les techniques actuelles pour l'étude expérimentale des structures $3 \mathrm{~d}$ des complexes (cristallographie, RMN, cryo-microscopie électronique, etc.) présentent de nombreuses limitations (taille, nature des protéines) et sont très coûteuses en temps et en prix. C'est pourquoi les techniques de docking ont été développées. Elles visent à prédire la structure $3 \mathrm{~d}$ fonctionnelle d'un complexe protéique à partir des molécules isolées, ce qui est considérablement plus facile à mettre en oeuvre, moins cher et plus rapide que les méthodes expérimentales in vitro (Grosdidier, 2007). Les approches utilisées actuellement sont exclusivement calculatoires et évaluées par des outils de visualisation. Ces approches peuvent être décomposées en quatre à cinq phases successives (figure 5] : (1) choix du mode de représentation des protéines (tout atomes, pseudo-atomes, grille, etc.), (2) exploration conformationnelle (corps-rigide position/orientation du ligand et/ou flexible position/orientation/forme du ligand), (3) minimisation de la fonction d'évaluation de l'énergie d'interaction (ou fonction de score) des conformations issues de l'exploration, (4) regroupement par ressemblances et classification par évaluation ou ré-évaluation plus fine du score, accompagnée d'une étape non automatique d'évaluation visuelle des résultats lorsque le score ne permet pas de discriminer la conformation native des différentes conformations générées et (5) une étape optionnelle d'affinement des complexes sélectionnés par minimisation ou dynamique moléculaire.

Un grand nombre des algorithmes de docking est fondé sur des approches exhaustives pour explorer l'espace de recherche conformationnel, dont le principal problème est l'explosion combinatoire du nombre de solutions. Ces approches peuvent être classées en trois familles : les approches dérivées d'échantillonnages systématiques, les techniques de la dynamique moléculaire, et les méthodes stochastiques. Elles ne produisent pas nécessairement des solutions correctes, et le temps de calcul s'avère être important. L'échantillonnage de l'espace de recherche doit être évalué à l'aide de fonctions objectives qui permettent de diriger cet échantillonnage, par le classement des modes d'interactions entre les protéines. La fonction objective idéale serait celle qui donnerait, pour un mode d'interaction donné, l'énergie libre liant deux protéines d'un complexe (voir section 2.2.2). Ces fonctions visent à reproduire les valeurs expérimentales des énergies libres de liaison et à atteindre par minimisation le minimum global pour des configurations de complexes protéiques.

Par conséquent, dans les cas concrets, les processus automatiques de docking doivent surmonter deux difficultés majeures pour parvenir à un résultat pertinent. La première est de traiter un espace de recherche dont la taille s'accroît considérable- 


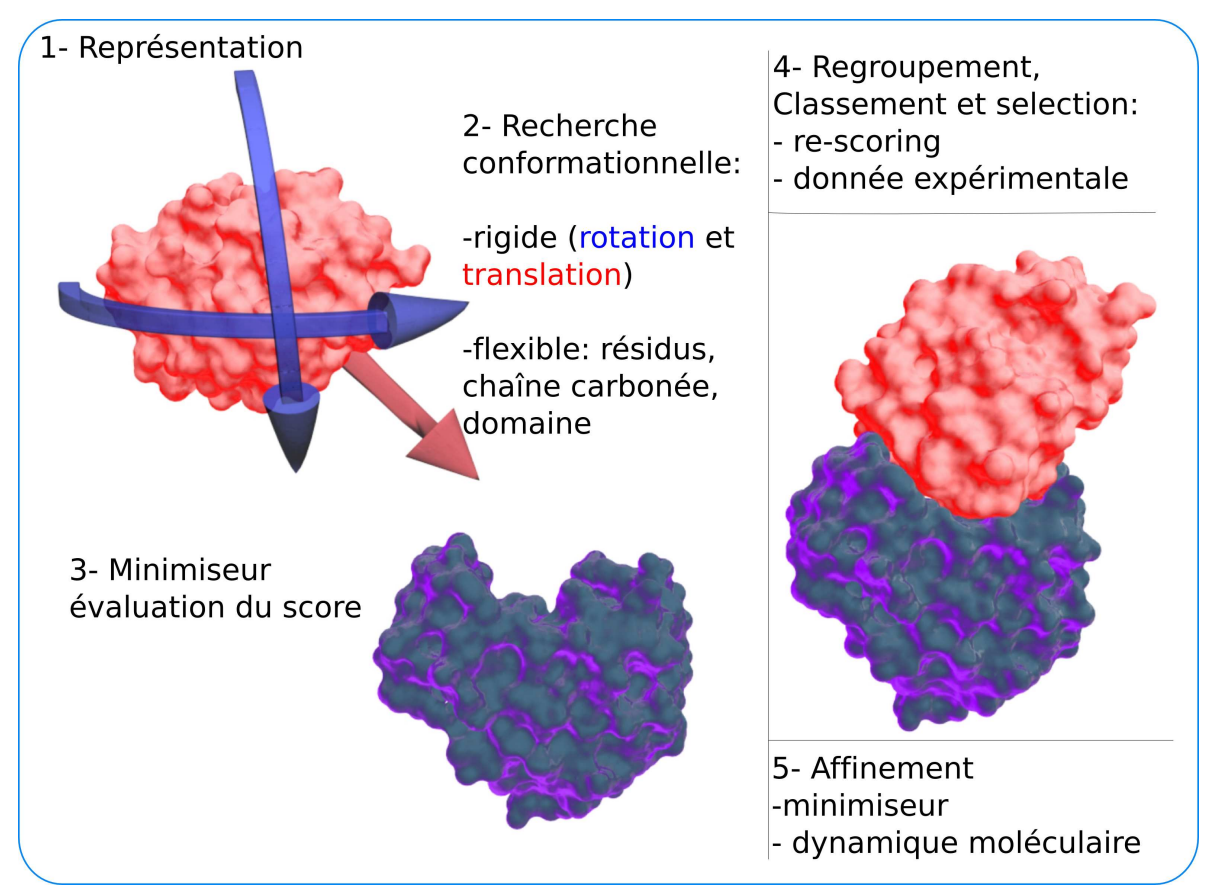

Figure 5. Schéma du protocole général de docking

ment avec le nombre de degrés de liberté décrivant la position et la conformation des protéines, et qui ne peut être traité dans un temps admissible. Le second problème est celui des algorithmes de recherche qui produisent des minima locaux et trouvent difficilement le minimum global normalement associé à la forme native du complexe (Wang et al., 2003).

Pour finaliser une simulation de docking, les chercheurs ont recours à une étape manuelle de visualisation moléculaire afin d'analyser les complexes générés. Cette activité réside en l'analyse en détail des résidus et atomes impliqués à l'interface de chaque complexe candidat en observant les liaisons hydrogènes, les ponts salins et surtout la présence de hot-spots (acides aminés situés à l'interface et qui sont connus pour être nécessaires à l'interaction). Toutefois, il est assez difficile de manipuler en même temps deux structures moléculaires $3 \mathrm{~d}$ et d'observer une interface (une protéine cache toujours l'autre) en utilisant des outils d'interaction classiques. C'est pourquoi le docking assisté par les techniques de RV multimodale présente une alternative pertinente pour améliorer l'activité du chercheur. Ces techniques permettent une interaction plus intuitive des structures protéiques $3 \mathrm{~d}$.

Enfin, concernant l'affinement des complexes sélectionnés, deux approches sont utilisées. Une première approche consiste à minimiser les corps rigides et les chaînes latérales des acides aminés à l'interface. Cette approche est implémentée dans de 
nombreuses applications : ICM-DISCO (Fernandez-Recio et al., 2003), FireDock (Andrusier et al., 2007), PELE (Borrelli et al., 2005), MMTK (Andrusier et al., 2000), ATTRACT (Zacharias, 2005), etc. L'autre approche consiste à étudier le comportement dynamique du complexe sélectionné. On citera le logiciel Gromacs (Hess et al., 2008) qui permet d'évaluer la position des atomes dans le temps en fonction des caractéristiques physico-chimiques. Cette approche permet d'une part d'évaluer la stabilité du complexe, et d'autre part d'évaluer les possibles changements conformationnels induits par l'interaction (déformation d'une boucle par exemple). Il faut néanmoins préciser que cette approche reste très coûteuse en temps de calcul par rapport aux minimiseurs qui sont pratiquement instantanés pour une configuration donnée.

\subsection{La Réalité Virtuelle et le docking}

Compte tenu des limites des algorithmes de docking classiques et des nouvelles possibilités offertes par la RV, des équipes se penchent depuis quelques années sur les problèmes liés au docking interactif et immersif. Les premiers travaux dans ce domaine ont porté principalement sur l'identification des besoins techniques et des limitations technologiques. Le système STALK (Levine et al., 1997) utilise des processus parallèles et distribués, d'une part pour le traitement du rendu visuel des modèles $3 \mathrm{~d}$ des protéines, et d'autre part pour les algorithmes de génération des solutions de docking possibles. Avec ce système, l'opérateur peut visualiser les deux protéines, assister l'algorithme de docking, le suspendre afin de pouvoir déplacer l'une des molécules en utilisant une souris $3 \mathrm{~d}$, et ensuite reprendre l'algorithme avec cette nouvelle position. Les évaluations de ce système ont porté sur la comparaison des énergies de liaisons moléculaires. Un autre système, le VRDD (Anderson et al., 1999) implémente un algorithme de recherche par la méthode Monte Carlo permettant d'affiner l'espace de recherche à travers l'exploration de solutions similaires. Les évaluations ont été effectuées sur trois cas de test, en comparant les valeurs de l'écart quadratique moyen (RMSD pour "Root Mean Square Deviation") entre une solution de docking et une structure cristallographique connue du même complexe protéique. Les résultats ont été jugés concluants pour deux cas de test, mais pas pour le troisième qui implique des protéines de grande taille (c'est-à-dire un grand nombre de solutions possibles) et flexibles (qui subissent des changements conformationnels). Ce travail a néanmoins permis d'identifier divers problèmes techniques liés au calcul en temps réel sur les énergies, la recherche et la construction des structures flexibles des protéines. Des travaux récents, comme celui de (Ray et al., 2005) et de (Ferey et al., 2008), se sont focalisés sur la résolution de ces problèmes par le renforcement du rendu visuel à l'aide d'un plugin qui interagit avec un programme de visualisation moléculaire hôte tel que Visual Molecular Dynamics (VMD). Le programme DockingShop (Lu et al., Aug 2005) semble être le plus avancé des systèmes de docking interactif. DockingShop implémente une représentation des protéines qui utilise les principes de la robotique, combinés à un affichage en temps réel des propriétés essentielles pour les interactions protéine-protéine (liaison hydrogène, énergie, complémentarité surfacique, interpénetration et effet hydrophobe). Plus récemment une approche robotique 
plus sophistiquée a été proposée pour la représentation des chaînes peptidiques et des molécules (Rossi et al., Jul 2007). Néanmoins, ces deux approches restent complexes quant à la quantité de données à transmettre à l'utilisateur et la limitation du type d'interface dont l'utilisateur dispose (souris/clavier et rendu visuel).

Des dispositifs plus avancés, tels que le tracking, ou les dispositifs haptiques utilisés en RV, permettent une manipulation directe et plus naturelle des objets dans un espace $3 \mathrm{~d}$. Ils semblent donc adaptés à la tâche de docking, puisque celle-ci consiste à évaluer des configurations définies par la position et l'orientation relative d'une protéine par rapport à l'autre. D'autre part, compte-tenu de la quantité importante d'informations nécessaires à l'utilisateur pour évaluer la qualité d'une configuration, il est pertinent de compléter le rendu visuel classique par d'autres rendus, à travers les deux autres canaux sensoriels que sont l'audio et l'haptique. Le retour haptique est connu pour améliorer la qualité d'interactivité de l'opérateur dans un environnement immersif, et sa perception des objets qu'il manipule (Seeger et al., 1997) ou des données qu'il analyse (Lundin et al., 2005). De même, les rendus audio peuvent améliorer la communication d'informations complexes (Barass et al., 2000). En outre, les substitutions et les redondances de ces rendus sur différents canaux peuvent avoir des effets bénéfiques sur les performances des utilisateurs, à condition que les modalités choisies soient pertinentes pour la tâche visée. Par exemple, (Richard et al., 2006) et (Kitagawa et al., 2005) ont montré que certains rendus audio et visuels peuvent communiquer efficacement des informations habituellement haptiques.

De nombreux projets ont examiné les possibilités offertes par ces retours multimodaux pour la tâche de docking. La plupart de ces projets utilisent des retours haptiques ou sonores mais rarement une combinaison des deux. Les projets orientés vers la modalité haptique sont principalement axés sur la génération du retour d'effort (force/couple) pour guider l'opérateur vers la meilleure solution. Ces projets incluent GROPE (Frederick P. Brooks et al., 1990), IVPS (Maciejewski et al., 2005) et SenSitus (Wriggers et al., 2003), un plugin de VMD qui permet à l'opérateur d'explorer les protéines avec un bras haptique. Ces projets visent à fournir des rendus simultanés de données volumiques multiples et à tester différents paradigmes d'interaction. Leurs plate-formes logicielles permettent de déplacer les protéines tout en ayant un retour haptique relatif aux collisions entre molécules. Les évaluations sont basées sur la mesure des erreurs de docking ainsi que sur les temps d'exécution pour différents paradigmes d'interaction (docking rigide vs flexible, visualisation stéréoscopique vs monoscopique, etc.). L'utilisation de la stéréoscopie permet de réduire le taux d'erreurs tandis que le retour haptique augmente le temps de manipulation en raison de l'exploration séquentielle et locale de la protéine. D'autres retours haptiques, tels que le retour vibrotactile, restent très peu appliqués. Concernant la modalité audio, les projets existants ont pour objectif de fournir à l'utilisateur des indices sur les propriétés moléculaires (en particulier sur les sites de fixation des protéines, la complémentarité de surfaces, etc.) en utilisant des earcons (icônes auditives) ou encore une sonification des données (Garcia-Ruiz et al., 2006). Divers travaux traitent de la sonification des données séquentielles telles que les séquences d'ADN (Hart et al., 2004) ou la soni- 
fication des complémentarités de surface entre protéines à partir de l'écart-type des distances minimales entre les couples d'atomes.

Au-delà des aspects purement techniques, le manque d'analyse des besoins de l'utilisateur, en amont de la réalisation de ces projets, produit au final un écart entre les résultats escomptés (des interactions naturelles avec des représentations de protéines générées par ordinateur et permettant un docking rapide et facile de complexes protéiques) et les résultats effectifs de tels projets (des systèmes techniquement impressionnants mais peu exploitables comme outils de travail). A l'opposé, notre approche a été d'impliquer les utilisateurs dans le processus de conception du système de docking de sorte à : (1) accélérer et faciliter le développement de prototypes fonctionnels à travers les choix de conception, (2) améliorer l'acceptation par les utilisateurs de ces nouvelles interfaces, et (3) proposer de nouvelles orientations non-explorées pour la recherche en docking et l'innovation (Anastassova et al., 2007). En outre, les outils de docking développés jusqu'à présent placent d'avantage l'utilisateur dans un rôle d'observateur ou de contrôleur des solutions, que dans celui d'un acteur majeur du processus. En d'autres termes, le chercheur ne fait pas partie intégrante du système et ne peut donc pas apporter son expertise par des interactions au cours même de la recherche de solutions. L'autre originalité de notre approche vise à intégrer le chercheur dans la boucle de docking.

Dans la section suivante, nous présentons donc une analyse de la tâche de docking, telle qu'elle s'exerce à l'heure actuelle, afin d'identifier les avantages et les inconvénients des outils existants. Ceci va nous permettre de déterminer les principes de base pour mettre en oeuvre des interactions multimodales en RV susceptibles de répondre aux objectifs décrits dans le paragraphe précédent.

\section{Analyse des besoins pour la conception d'un Environnement Virtuel Multimodal dédié au docking}

\subsection{Méthode}

Notre étude s'est d'abord focalisée sur l'analyse de l'usage des outils de docking existants, conçus pour des interfaces de bureau, ainsi que de l'impact pressenti, par les utilisateurs, de l'introduction de technologies de RV et de solutions multimodales pour cette activité. Quatre chercheurs en bioinformatique, âgés de 28 à 50 ans (moyenne=36 ans, ET=9,95), ont pris part à cette expérience. Chacun utilise des logiciels de docking dans le cadre de son travail quotidien, avec en moyenne 5 ans d'expérience dans leur utilisation.

Nous avons utilisé deux techniques pour le recueil de données : des entretiens semi-dirigés et l'observation de l'activité sur le lieu de travail. En premier lieu, nous avons réalisé, enregistré et retranscrit verbatim quatre entretiens sur le lieu de travail. Ces entretiens étaient confidentiels et anonymes. Ils s'appuyaient sur un guide composé de plusieurs questions portant sur des sujets divers tels que le type de problèmes de docking auxquels le chercheur était confronté dans le cadre de son travail, le type 
de logiciels utilisés, les difficultés couramment rencontrées, ainsi que la manière dont les chercheurs envisageaient de travailler dans un Environnement Virtuel Multimodal (EVM). Ces entretiens furent soumis à une Analyse Cognitivo-Discursive (ACD) (Ghiglione et al., 1998) réalisée à l'aide du logiciel Tropes (développé par Acetic Software). L'analyse se focalisait sur le thème des interactions protéine-protéine, et visait à identifier les éléments (comme les types de données, etc.) impliqués dans l'évaluation d'une telle interaction. En second lieu, nous avons enregistré puis analysé trois séances de travail sur des logiciels de docking standard : ICM-Disco (Fernandez-Recio et al., 2003), ClusPro (Comeau et al., 2004), et Hex (Ritchie, 2003). Les données vidéo ont servi de base à une analyse de la tâche de docking. Les protocoles verbaux ont été recueillis tout au long de la séance et analysés en prenant pour unité d'analyse la verbalisation explicite d'une tâche. Le codage des actions et des verbalisations recueillies dans ces séances de travail a permis d'élaborer deux ressources pour la conception : un modèle de la tâche sous forme d'une décomposition hiérarchique, basé sur la méthodologie du Hierarchical Task Analysis (HTA) (Annett, 2003) et un résumé illustré du déroulement de la séance sous forme d'un storyboard décrivant les tâches réalisées dans le cadre de la résolution d'un problème de docking type : le docking du complexe Barnase / Barstar.

\subsection{Résultats}

Les résultats de l'analyse nous ont permis de construire trois éléments susceptibles d'assister la conception d'un EVM pour le docking moléculaire : (1) un modèle des tâches réalisées, (2) un modèle des besoins de l'utilisateur et (3) un ensemble de principes pour guider les choix en matières de distribution des informations sur différentes modalités sensorielles et de conception des affichages et des techniques d'interaction correspondants.

\subsubsection{Analyse hiérarchique de la tâche}

L'arbre des tâches HTA (figure 6) décrit une manière de décomposer l'activité de docking en fonction des objectifs à atteindre. En résumé, une première tâche est de générer un ensemble de grande taille de modèles potentiels du complexe protéique. En entrée de cette tâche sont utilisés des fichiers décrivant la structure $3 \mathrm{~d}$ des protéines dans leur forme non-liée, stockée dans la $P D B$. La seconde étape consiste en l'exécution d'un algorithme automatique d'exploration de l'espace conformationel associé à une évaluation d'énergie (score) pour éliminer les solutions physiquement impossibles, et à du clustering pour classer les solutions restantes par similarité (clusters). Les complexes obtenus sont ordonnés en fonction de leur score. Enfin, le chercheur sélectionne un petit nombre d'entre elles comme candidates pour l'étape de validation expérimentale. 


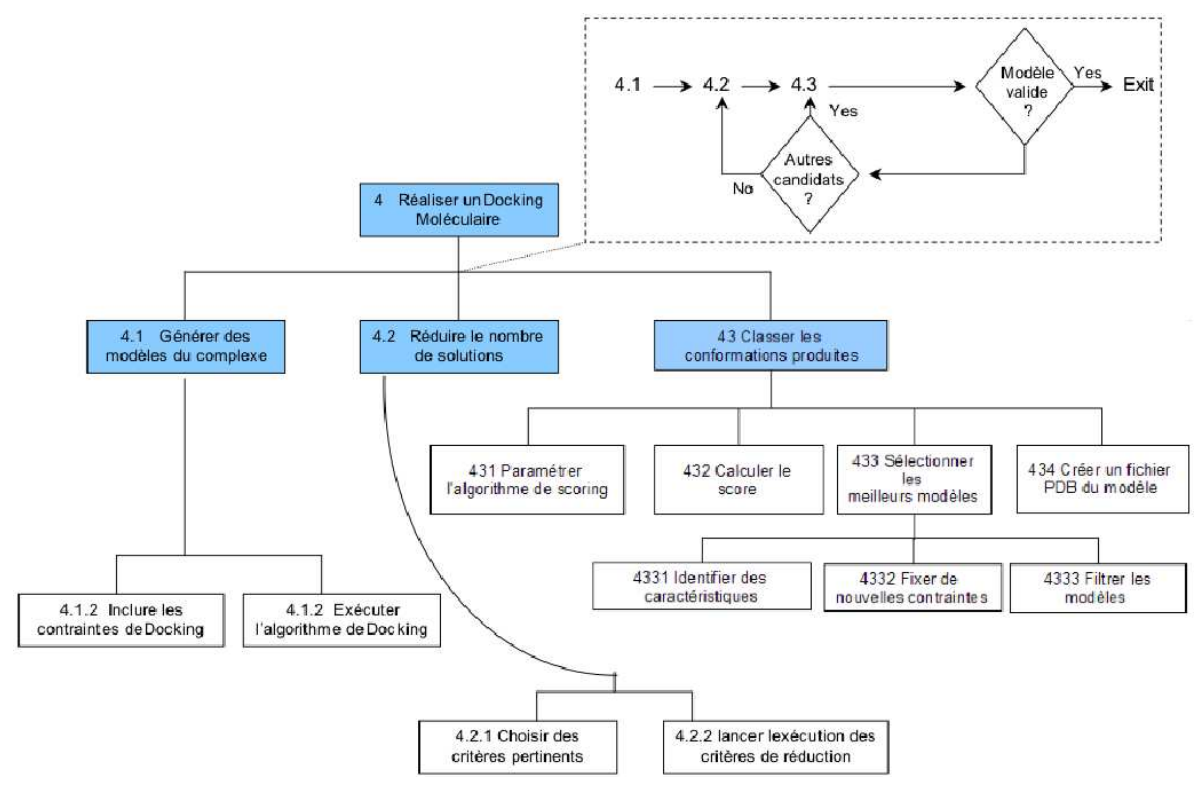

Figure 6. Vue partielle de l'arbre des tâches HTA pour le docking moléculaire et planification de ces tâches (encadré).

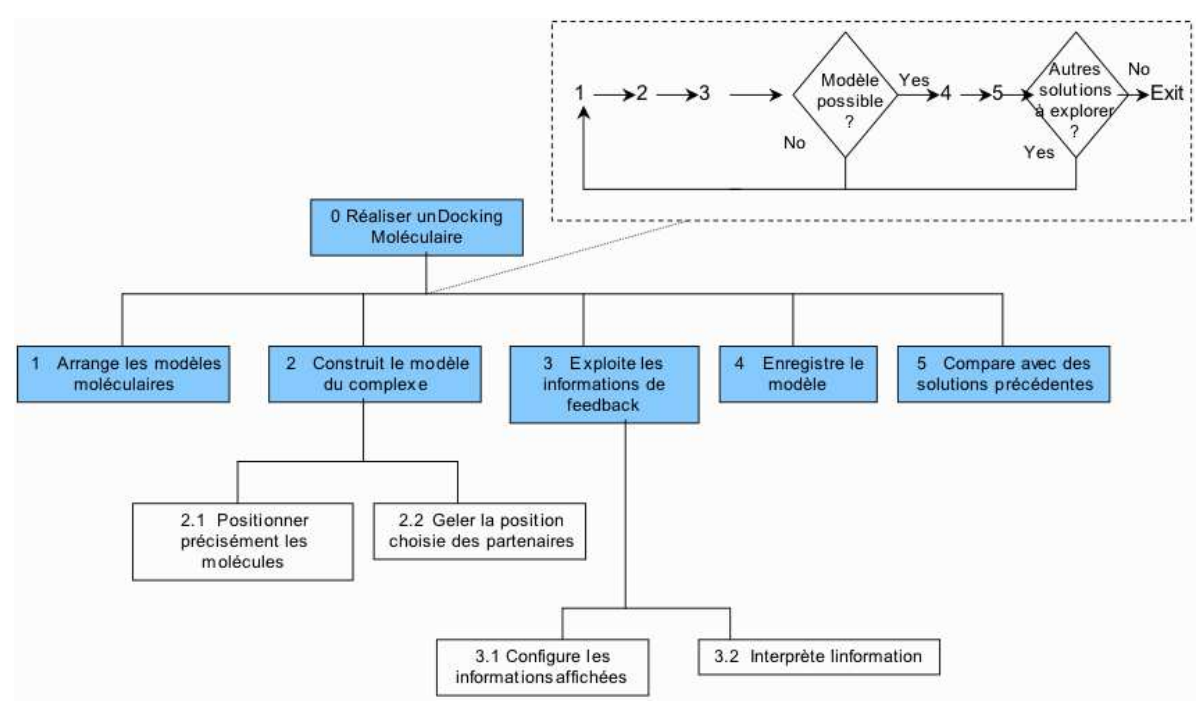

Figure 7. Proposition d'un arbre des tâches HTA pour le docking réalisé dans un contexte multimodal et planification de ces tâches (encadré). 


\subsubsection{Besoins en termes de présentation d'informations}

L'ACD a mis en relief, au travers des pondérations et des proximités géométriques entre les référents centraux du discours des chercheurs, quatre besoins de base en information concernant le docking. Les trois premiers ont trait aux propriétés des molécules étudiées dont l'interaction est perçue comme centrale dans la recherche d'une conformation et de son ajustement :

- La complémentarité topologique des molécules ( $c f$. section 2.2.1);

- Les caractéristiques énergétiques ( $c f$. section 2.2.2);

- Les patches et interactions hydrophobes ( $c f$. section 2.2.5) ;

- Le quatrième besoin concerne les propriétés déjà connues de l'interface moléculaire considérée, lorsque celles-ci sont disponibles. Il s'agit de la liste d'acides aminés dits hot-spots dont le biologiste sait qu'ils participent à l'interaction et sont donc présents à l'interface.

Il est intéressant de noter que le poids statistique de la fonction de score dans l'ACD était nul. Ceci suggère que cette fonction de score n'est pas considérée comme un outil incontournable du docking mais plutôt comme un moyen parmi d'autres. Ce qui est cohérent avec la théorie en amont de la fonction de score. En effet, nous avons vu que le score était évalué à partir d'équations approximant les phénomènes physicochimiques en vue de refléter au mieux l'énergie d'interaction. Néanmoins dans la majorité des cas, ces fonctions de score ne permettent pas de discriminer la bonne conformation parmi les différents modèles générés. Les entretiens ont montré que le docking implique l'étude conjointe de ces trois propriétés moléculaires en fonction des connaissances préalables sur les propriétés de l'interface. L'observation des sessions de travail montre d'ailleurs parallèlement que les chercheurs passent en moyenne $42 \%$ de la durée des séances de travail à consulter des sources de données externes telles que la base de données PubMed ou bien des notes de travail, en vue de trouver les coordonnées de ces hot-spots.

\subsubsection{Critères et recommandations concernant l'allocation modale}

Le terme d'allocation modale (André, 2000) désigne l'utilisation spécifique d'une ou plusieurs modalités pour présenter une information. Il est souhaitable pour l'utilisateur de prévoir une allocation modale optimale compte-tenu des contraintes à la fois techniques (ex. dispositifs de RV), liées à la tâche de docking (ex. caractéristiques des informations et intérêt pour le chercheur) ou encore humaines (ex. caractéristiques perceptives, expertise, etc.).

Proposer des principes d'allocation modale implique de donner du poids à certaines associations information-modalité en fonction des contraintes. Un problème est que l'on ne dispose pas a priori de spécifications ergonomiques concernant l'allocation modale des informations centrales pour ce type de tâche. En effet, bien que la recherche sur la conception d'Interfaces Homme-Machine multimodales ait débuté il y a plusieurs décennies, rares sont les travaux qui proposent des principes robustes pour choisir telle modalité de présentation pour tel type d'information. D'autre part, 
peu de travaux portent sur la conception d'IHM pour les activités d'exploration de données. Il est cependant possible de formuler des hypothèses en s'appuyant d'une part sur la façon dont les sujets humains traitent l'information et, d'autre part et encore plus fortement, sur les exigences mises en évidence par l'analyse de l'activité. Dans le cas du docking moléculaire, cette dernière a montré un besoin de présenter de une à quatre sources (au plus) d'informations simultanément suivant l'étape et les connaissances préalables disponibles : la topologie moléculaire, l'hydrophobicité des résidus, les champs électrostatiques et les résidus potentiellement impliqués dans l'interface (voir section précédente).

L'analyse de l'activité a permis d'identifier également les contraintes suivantes, liées à la tâche de docking :

- Les quatre types d'informations pouvant être utilisés par le chercheur à un instant donné, il est nécessaire d'anticiper un risque de surcharge si ces informations sont présentées simultanément sur le même canal et selon des modes proches. Il en résulte que les informations doivent être réparties de façon équilibrée entre les différents canaux sensoriels pour éviter une telle surcharge, en particulier visuelle. Bien que la sonification et l'haptisation soient susceptibles de décharger efficacement le canal visuel et d'améliorer les performances, une répartition aléatoire des informations sur les différentes modalités disponibles pourrait aboutir à des performances inférieures à celles sur une interface où l'ensemble de l'information serait concentrée sur un seul mode. Il est donc nécessaire de se doter de critères pour décider des associations a priori les plus acceptables du point de vue de l'utilisateur.

- Une distinction importante de ce point de vue concerne le statut invariant et pérenne versus dynamique et transitoire de l'information au cours de la session de docking. Certaines informations, telles que la structure moléculaire, doivent être disponibles en permanence, et ne se modifient pas au cours d'un épisode de l'activité. Si l'on choisit de fournir un rendu auditif ou haptique de ces informations qualifiées de pérennes, une conséquence des propriétés du système cognitif de traitement de l'information pourrait être le filtrage perceptif de ces informations, c'est-à-dire leur disparition du foyer attentionnel ; une autre conséquence pourrait être une gène pouvant aller jusqu'à la désactivation des fonctions de présentation correspondantes par l'utilisateur, du fait qu'il juge l'information acquise et trop invasive. Pour les informations pérennes, nous proposons par conséquent d'utiliser la modalité visuelle.

- Un autre critère de choix concerne la sémantique de l'information et sa proximité plus ou moins grande avec l'une ou l'autre des modalités candidates. Comme nous l'avons dit, la prise en compte d'informations telles que les forces électrostatiques ou les interactions hydrophobes est indispensable pour la construction des complexes de protéines. Les forces électrostatiques pourraient être présentées à l'aide d'une contrainte haptique car cette modalité peut véhiculer des informations d'attraction ou de répulsion d'une manière qui évoque des situations de la vie quotidienne. Les modalités visuelle et auditive ne doivent pas être éliminées d'office pour autant; mais leur usage impliquerait d'élaborer une échelle continue où la distinction attraction - répulsion serait apparente, ainsi que le "point zéro" de l'échelle (ex. signal à 1 


\begin{tabular}{|l|c|c|c|}
\hline & Visuelle & Auditive & Haptique \\
\hline Représentation protéine (surface) & $\checkmark$ & & \\
Collisions (non interpénétration) & $\checkmark$ & $\checkmark$ & $\checkmark$ \\
Score de complémentarité géométrique & $\checkmark$ & $\checkmark$ & $\checkmark$ \\
\hline Interactions énergétiques & $\checkmark$ & $\checkmark$ & $\checkmark$ \\
\hline Interactions hydrophobes & $\checkmark$ & $\checkmark$ & $\checkmark$ \\
\hline Connaissances a priori (hot-spots) & $\checkmark$ & $\checkmark$ & \\
\hline
\end{tabular}

Tableau 1. Restriction de l'espace des allocations modales

ton pour attraction et 1 ton pour répulsion).

- Un troisième critère découle de l'existence d'une organisation à deux niveaux de l'activité d'exploration : le modèle HTA de l'activité prospective (figure 7) montre que les utilisateurs souhaiteraient réaliser le docking en deux étapes. Comme le montrent les entretiens, la première étape consisterait à identifier une zone potentielle d'interaction en examinant la protéine au niveau moléculaire (global). Elle implique la mise en oeuvre de connaissances préalables sur l'interface, de critères topologiques, et l'identification de patchs hydrophobes, c'est-à-dire des aires étendues de résidus hydrophobes. La seconde étape, au niveau atomique (résidus) vise à évaluer la compatibilité entre deux zones de contact potentielles, susceptibles donc de se retrouver en contact à l'interface. Elle implique un examen plus détaillé de l'interaction, c'està-dire des interactions hydrophobes, électrostatiques, etc. A mesure qu'il teste ces différentes possibilités d'interaction, le chercheur devrait disposer d'informations sur des zones d'intérêt plus précises (nature des liaisons chimiques à l'interface, patchs hydrophobes, champ électrique, hot-spots, etc.), mais aussi sur le complexe résultant de l'interaction testée (score).

Ces contraintes nous permettent d'énoncer les principes suivants pour la conception d'une application multimodale pour le docking, résumés dans le tableau 10:

- Utiliser au moins la modalité visuelle pour afficher les surfaces moléculaires, pour permettre la manipulation des modèles des protéines ;

- Présenter simultanément toutes les informations impliquées dans le calcul des énergies de liaison, à l'aide d'une combinaison de modalités ;

- Rester si possible proche d'expériences perceptives courantes de la vie quotidienne et cohérentes avec la sémantique des informations (ex. faire un rendu haptique des collisions et des forces électrostatiques);

- Les signaux auditifs peuvent être utilisés pour sonifier des variables variant dans le temps, telles que le score, la présence de hot-spots à l'interface, la présence de liaisons hydrogène à l'interface, etc. ;

- Utiliser une combinaison de modalités pour présenter la plus grande quantité possible d'informations simultanées sans surcharger cognitivement l'utilisateur. 
Il est important de souligner que ces principes sont en partie des hypothèses à valider notamment dans le cadre des évaluations mises en oeuvre dans le projet. Ces dernières, en cours, sont évoquées dans la section 7 .

\section{Une approche hybride et multimodale du docking}

\subsection{Réconcilier les approches automatiques et interactives}

Notre approche a pour objectif de combiner les apports des rendus multimodaux et les connaissances des experts en docking dans certaines phases de l'étude. En permettant à l'utilisateur d'interagir au plus tôt dans le processus plutôt qu'a posteriori ou par unique paramétrage du système, nous espérons réduire d'une part les temps de traitement et d'autre part les risques de faux positifs. Plus précisément, le protocole que nous développons comporte trois étapes (figure 8). Une première étape en environnement immersif, nommée Réduction de l'espace de recherche, permet à l'utilisateur biologiste de pré-positionner le système dans les quelques configurations qui lui semblent intéressantes grâce à des rendus visuels, sonores et haptiques. Cette première étape permettra de réduire rapidement l'espace de recherche en s'appuyant sur les capacités d'analyse de motifs 3d de l'expert et sur ses connaissances spécifiques en docking de protéines. Les données sur les interactions à ce stade sont approximées ou qualitatives de manière à conserver l'interactivité, les contraintes les plus fortes se situant notamment au niveau des retours haptiques. Une deuxième étape sera apparentée à la procédure automatique de docking classique mais uniquement sur les espaces restreints ainsi définis par le biologiste. Cette étape consiste à évaluer un score plus fin pour les conformations sélectionnées lors de la première étape ainsi que les soumettre à une phase automatique d'affinement (minimisation en corps rigide). Et enfin, la troisième étape, appelée Exploration du classement, s'effectue de nouveau en environnement immersif et permet au chercheur d'explorer de manière multimodale les solutions de la première étape et de les trier en fonction de la deuxième étape afin d'extraire celles qu'il désire tester de manière expérimentale. Dans cette étape le besoin de précision est plus important mais les déplacements entre molécules, à l'origine de calculs complexes, peuvent être bloqués.

\subsection{Architecture matérielle et logicielle}

L'architecture matérielle et logicielle de notre environnement de docking multimodal est fondée sur une plate-forme de RV client/serveur. L'immersion visuelle est assurée par un dispositif de type CAVE (Cruz-Neira et al., 1992) doté d'un système de rétro-projection à stéréoscopie active. Les retours sonores $3 \mathrm{~d}$ sont générés avec MaX/MSP, la spatialisation des sons est assurée par SPAT et leur diffusion se fait soit par un casque, soit par un ensemble de huit haut-parleurs répartis dans le dispositif immersif. Le rendu haptique, quant à lui, est assuré par le bras haptique Virtuose commercialisé par Haption ${ }^{\circledR}$. La capture des mouvements de l'utilisateur - ceux de 

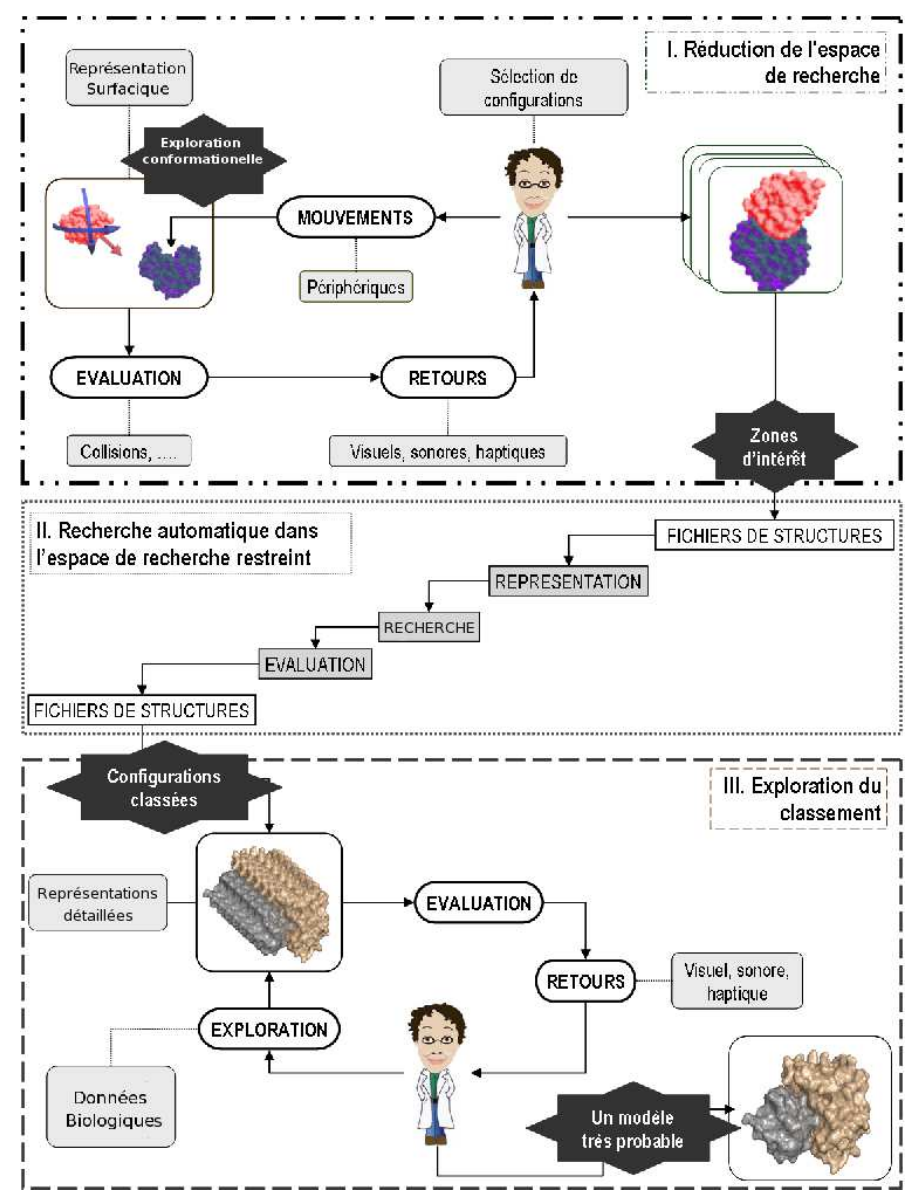

Figure 8. Notre approche hybride et multimodale du docking.

sa tête pour la stéréoscopie adaptative et ceux de sa main manipulant une souris $3 \mathrm{~d}$ - est assurée par le système de caméras et de capteurs infrarouges ARTrack ${ }^{\circledR}$. Tous ces périphériques sont gérés par des clients indépendants. La distribution des événements et des données entre ces clients (figure 9 ) est assurée par la plateforme logicielle VEserver (Touraine et al., 2002). Chaque client possède sa propre représentation des protéines et de leurs caractéristiques, adaptée au périphérique concerné. Les différents rendus (visuels, haptiques ou sonores) des deux protéines manipulées sont synchronisés par l'encapsulation de la position et de l'orientation de chaque protéine dans des messages UDP, encapsulation utilisant le protocole Open Sound Control.

\subsubsection{Modalités visuelles}

Afin de respecter l'expérience quotidienne des chercheurs et pour ne pas re- 


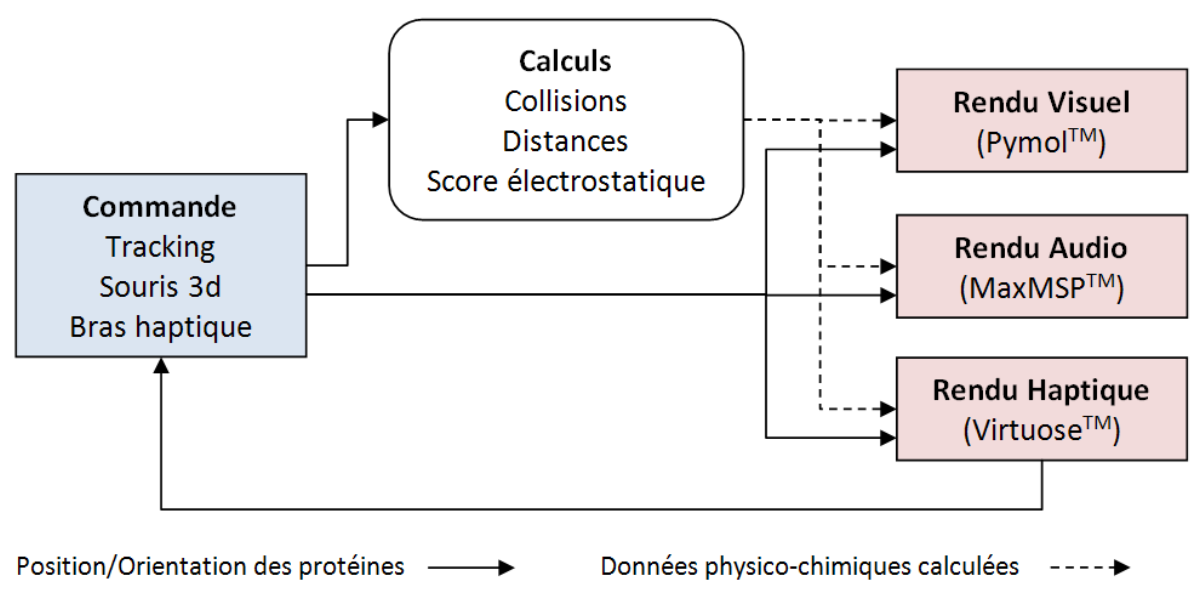

Figure 9. Architecture de la plateforme dédiée au docking multimodal immersif

développer l'ensemble des représentations visuelles usuelles, nous avons choisi de ré-utiliser un des nombreux outils de visualisation moléculaire. Notre choix s'est porté sur le logiciel libre Pymol, qui offre les différentes modalités visuelles nécessaires à l'activité de docking :

- la représentation surfacique, obtenue d'après des méthodes variées, selon la granularité de surface désirée : surface accessible au solvant (Sanner et al., 1996), surface moléculaire (Connolly, 1983b) ;

- la représentation atomique : (i) de type CPK (Corey et al., 1953) représentant les atomes comme des sphères d'un rayon égal à leur rayon de Van der Waals, (ii) de type bâton ne représentant que les liaisons covalentes par un tube ou une ligne, (iii) de type boule-bâton représentant le noyau des atomes par des sphères et les liaisons covalentes par un tube, et (iv) de type fils de fer ne représentant que les liaisons entre les atomes par un trait ;

- la représentation de la structure secondaire (hélice $\alpha$ et feuillet $\beta$ ) : de type ruban (un tube uniforme suit la chaîne carboné) ou de type cartoon (les hélices et les brins sont schématisés par des flèches).

De ces trois grands types de représentation, toutes les combinaisons sont possibles afin de visualiser une protéine. De plus, sur ces représentations peuvent être projetées des informations diverses, comme la nature des atomes et des acides aminés à la surface, les charges portées par ces atomes, les propriétés d'hydrophobicité, le degré de flexiblité, la conservation ou encore les hot-spots.

Les autres critères de ce choix logiciel ont été :

- la maîtrise par les utilisateurs experts ; 


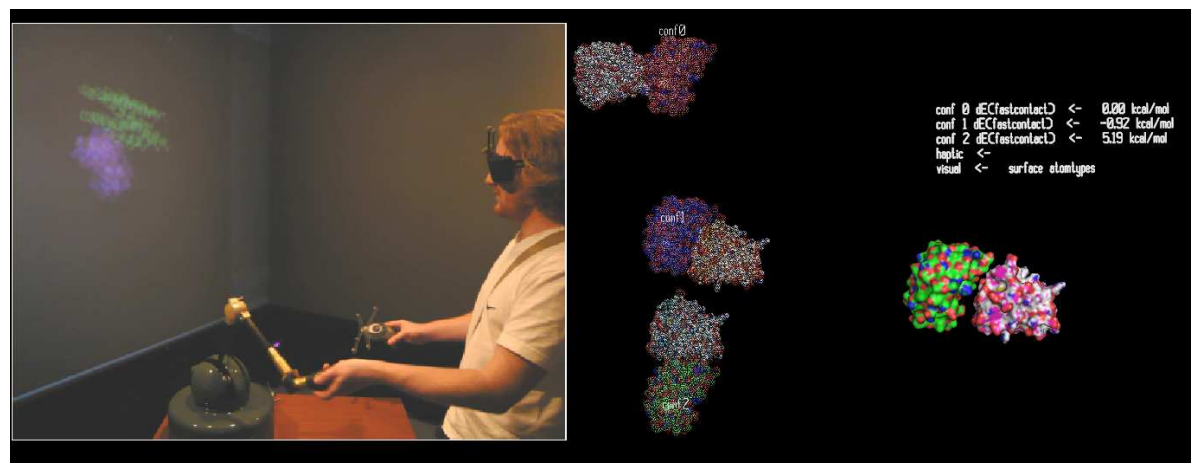

Figure 10. Utilisateur immergé dans l'application du docking, à droite un exemple de capture d'écran aprés la selection de 3 conformations par l'utilisateur.

- la possibilité d'intégrer des données particulières, obtenues a priori dans la bibliographie (comme les hot-spots), ou implicites à l'expertise de l'utilisateur ;

- l'existence d'une API programmable (ici Python) permettant d'ajouter rapidement des fonctionnalités visuelles ou interactives (en l'occurence sous OpenGL);

- un grand nombre de plug-in ou scripts disponibles et utiles à notre application.

\subsubsection{Manipulation bimanuelle}

Afin de manipuler les deux protéines et de tenter de réaliser un docking virtuel, l'opérateur dispose de plusieurs dispositifs et de plusieurs paradigmes de manipulation (figure 10). Un premier paradigme associe la position et l'orientation d'une protéine, la plus petite (le ligand), à celle d'un bras à retour d'effort, la position de la seconde protéine (la plus grosse, récepteur) étant fixe. L'orientation de la protéine récepteur est contrôlée par un trackball disponible sur le bras ou sur la souris 3d. Cette modalité correspond à un usage, puisque dans les solutions de docking, la protéine récepteur est fixe et ce sont la position et l'orientation du ligand qui sont explorés. L'autre paradigme, plus naturel, consiste à manipuler une protéine avec le bras à retour d'effort, et l'autre avec la souris $3 \mathrm{~d}$, l'orientation et la position de chacune étant asservies à celles de ces dispositifs. Cette modalité est symétrique en terme de manipulation, c'est-àdire en terme de commande, mais n'est pas symétrique en terme de rendu, puisque le rendu haptique n'est disponible que pour une des deux protéines.

\subsubsection{Modalités haptiques}

A l'heure actuelle, il existe réellement peu de systèmes de docking moléculaire intégrant à grande échelle un retour haptique (force/tactile). Ceci est principalement dû à la complexité des calculs des moteurs physiques utilisés pour la simulation de la dynamique moléculaire, qui rend difficile le respect des contraintes de rafraichissement haptique temps réel $(200 \mathrm{~Hz}-1 \mathrm{kHz})$. Cela nous a conduit à ne considérer dans 
notre premier système que des protéines rigides, pour un docking de type clé/serrure ou $L E G O$.

Plusieurs rendus haptiques ont été implémentés pour rendre les collisions et les phénomènes énergétiques. Dans tous ces rendus haptiques, une des deux protéines est attachée au dispositif haptique, et l'autre à la souris $3 \mathrm{~d}$, ce qui permet de manipuler leurs positions et leurs orientations par rapport à leurs barycentres.

Collision - La détection de collision s'effectue sur un client graphique basé sur des applications $C++$ /Open $G L$ afin d'assurer une cohérence visuelle entre la représentation haptique des protéines, et leur représentation visuelle dans l'environnement de visualisation Pymol. Pour chaque protéine, un maillage de la surface est d'abord précalculé en utilisant MSMS (Sanner et al., 1996). La résolution de ce maillage peut être paramétrée. Ensuite, la routine de détection de collision utilise la librairie RAPID (Gottschalk et al., 1996) qui permet de calculer en temps interactif la liste des triangles en collision entre les deux maillages durant le docking. Pour le rendu haptique, deux méthodes de calculs des forces ont été implémentées :

La première consiste à rendre une force de répulsion dont la direction est définie par les barycentres des deux protéines en collision, et dont le module est proportionnel au nombre de triangles en collision. Lorsqu'un rendu en torsion est possible, le couple est défini par la résultante des normales des triangles en collision et le barycentre de la protéine attaché au dispositif haptique.

La seconde approche, plus complexe mais plus pertinente, consiste, à partir des triangles en collision, à calculer une distance ou un volume d'interpénétration (au lieu du nombre de triangles en collision), qui pondère la force de répulsion.

Caractéristiques électrostatiques - Deux approches sont proposées pour permettre à l'utilisateur de ressentir les phénomènes électrostatiques.

La première approche consiste à calculer les forces à appliquer à chaque particule chargée à l'aide de l'équation de Coulomb ( $c f$. section 2.2.4 figure 4), en considérant chaque couple de particules chargées. La complexité de ce calcul est quadratique, mais reste appropriée dans le cas de protéines de tailles moyennes, le nombre de particules chargées dans une protéine étant limité.

Dans la seconde, le champ global du potentiel électrostatique de la protéine cible (récepteur) est calculé a priori en utilisant $A P B S$ 2, qui permet d'obtenir une grille de potentiel électrostatique $3 \mathrm{~d}$ en résolvant l'équation de Poisson ( $c f$. section 2.2.4). Le gradient du potentiel électrostatique permet d'obtenir le vecteur du champ de force pour chaque cellule de la grille $3 \mathrm{~d}$. Les acides aminés chargés de la seconde protéine (ligand) sont ensuite immergés dans ce champ de force électrostatique $3 \mathrm{~d}$ autour du récepteur. Cette méthode nous permet de calculer une force d'interaction électrostatique pour chaque particule en temps linéaire en fonction du nombre de particules chargées qui constituent le ligand.

2. Adaptative Poisson-Boltzmann Solver (Baker et al., 2001) 
Interactions de Van der Walls - La force de Van der Walls entre deux atomes est calculée selon les équations de la section 2.2.3. Contrairement aux forces électrostatiques, ces forces s'appliquent à très courte distance, et ne concernent donc que les atomes de surface. Les résultantes translationnelle et rotationnelle des forces de Van der Walls sont calculées sur chaque particule, appliquées au barycentre du ligand et ajoutées aux forces électrostatiques, ce qui permet à l'utilisateur de ressentir une complémentarité énergétique globale entre les deux protéines.

Nous pouvons noter qu'au niveau perceptif, le rendu des interactions de Van der Walls est semblable à celui des collisions de surface, puisqu'il prévient l'interpénétration. Toutefois, cette information est quantitative et biologiquement pertinente, et non plus seulement géométrique et qualitative.

\subsubsection{Modalités sonores}

De manière complémentaire, certaines informations peuvent être diffusées via l'audio, si les canaux visuels et/ou haptiques sont déjà utilisés pour rendre d'autres paramètres. Par ailleurs, le canal auditif est particulièrement adapté pour rendre des paramètres globaux. Certains de ces paramètres globaux sont souvent difficilement visualisables (ex. l'interface du complexe n'est pas visible si l'on n'écarte pas les deux protéines, ou si l'on ne modifie pas la modalité d'affichage du ligand). C'est le cas des paramètres suivants :

- Score global de complémentarité géométrique de surface : cette complémentarité est calculée à partir de l'écart-type des distances minimales. Pour chaque atome de surface de la première protéine, on calcule l'atome de surface le plus proche dans la seconde, ce qui nous donne un ensemble de distances minimales. Plus ces distances sont homogènes, plus la complémentarité de surface est bonne.

- Energie électrostatique globale du complexe courant : cette énergie est calculée à partir des énergies d'interactions electrostatiques entre chaque particule chargée ( $c f$. section 2.2.4.

- Nombre de liaisons hydrogène potentielles entre les acides aminés à l'interface du complexe : lorsque deux atomes de surfaces capables de créer une liaison hydrogène sont suffisamment proches (distance inférieure à $2.5 \AA$ ), il y a une liaison hydrogène potentielle. Ce nombre de liaisons à l'interface est important pour évaluer la qualité du complexe.

- Nombre de hot-spots à l'interface du complexe : il s'agit de la liste des acides aminés à l'interface, déterminés de manière expérimentale comme participant à l'interaction. Pour évaluer la qualité du complexe, le fait que ces hostpots se situent à l'interface des deux protéines est important.

Plusieurs paradigmes de sonification de données ont donc été conçus, pour rendre individuellement ou simultanément plusieurs de ces paramètres :

- Le premier, qui permet de ne rendre qu'une seule mesure, est l'encodage d'une mesure scalaire par un déphasage aléatoire d'un son identique superposé plusieurs fois. Ce déphasage aléatoire est inversement proportionnel à la qualité de la mesure. Il 
permet de rendre un son pur ou chaotique, dans le cas d'une sinosoïde, ou de rendre un mot compréhensible ou non, dans le cas d'un mot pré-enregistré, selon que la mesure est bonne ou mauvaise. Ce paradigme est particulièrement adapté lorsque l'on connait a priori l'optimum de la mesure, et que l'on ne dispose pas de spatialisation sonore.

- Le second paradigme permet de rendre simultanément deux mesures par encodage à la fois de la tonalité et de l'amplitude de ces deux mesures. Il est particulièrement adapté lorsque l'échelle de grandeur de ces mesures est connue a priori, mais que l'optimum n'a pas vraiment de sens.

- Le troisième, plus complexe car il nécessite l'utilisation de la spatialisation, permet de rendre simultanément plusieurs mesures. Il consiste à faire la correspondance entre la localisation fixe d'une source sonore dédiée au rendu d'une mesure, à la sémantique de cette mesure. Chaque zone de l'environnement spatial auditif est donc consacrée au rendu d'une seule mesure.

\subsubsection{Superviseur multimodal}

Nous avons vu qu'une partie des choix de distribution multimodale est réalisée de manière statique, en amont de l'exécution de l'application de docking, grâce aux recommandations ergonomiques issues de l'analyse des tâches. Cependant, la réalisation des rendus (ou "instanciation") doit être contrôlée tout au long de l'application. Ceux-ci doivent dépendre des caractéristiques ponctuelles des données mais aussi du contexte des interactions. Ce contexte est lié à l'utilisateur, à l'environnement réel, à l'environnement virtuel et aux dispositifs.

Pour effectuer cette gestion dynamique des rendus multimodaux, nous avons modélisé et développé un processus de supervision (Bouyer, 2007) (Bouyer et al., 2008). Nous l'avons d'abord conçu de manière générique, adaptable à différentes applications, avant de le spécialiser pour les besoins de la tâche de docking.

Cette supervision repose sur quatre acteurs principaux (figure 11) :

Le monde réel - Le biologiste commande le système à l'aide de la souris $3 \mathrm{~d}$ et du bras haptique, et perçoit les rendus multimodaux par l'intermédiaire des écrans, des haut-parleurs et du même bras haptique. L'utilisateur est également suivi via différents capteurs (tête, main, etc.).

L'application - Nous modélisons l'application de docking comme un trio d'éléments. L'Environnement Virtuel (EV) contient une représentation virtuelle de l'utilisateur, ainsi que l'ensemble des données qui vont faire l'objet des interactions : protéines, scores, variables numériques, etc. L'application est également soutenue par une architecture logicielle et matérielle. Les interfaces d'entrée et de sortie sont dirigées par des pilotes et les rendus qui y sont présentés sont générés par des moteurs de rendu (graphique, sonore et haptique). Enfin, le gestionnaire d'interaction interprète les commandes de l'utilisateur, modélise les interactions, demande au superviseur de contrôler le rendu et commande à l'architecture de présenter le rendu décidé. 
Le superviseur - Le superviseur est en charge du contrôle du rendu multimodal. Pour cela, il détient une base de connaissances renfermant les règles de distribution des modalités ( $c f$. recommandations ergonomiques), une base de règles de décision qui déterminent son comportement et une base de contexte qui contient les éléments susceptibles d'influer sur le rendu.

L'observateur/interpréteur - Ce module permet la communication entre l'application de docking et le superviseur, en faisant office de traducteur des informations échangées. Son second rôle est d'observer en temps réel l'exécution de l'application afin d'en isoler les éléments du contexte et de les mettre dynamiquement à disposition du superviseur. Les éléments du contexte peuvent être issus du monde réel (comme les données de suivi) et informer par exemple de la position de l'utilisateur ou de ses mouvements, ou bien de l'EV et informer de l'organisation spatiale de la scène (position relative des deux protéines, etc.). La connaissance des capacités de rendu de l'application sont particulièrement utiles : les médias et les modalités disponibles dans l'application, les charges dynamiques des canaux sensoriels, etc.

De manière plus détaillée, le processus de supervision consiste en un échange successif de messages entre ces quatre acteurs :

- Dans le cas le plus simple, l'utilisateur indique au système qu'il désire réaliser une interaction sur les données de l'environnement virtuel (principalement les protéines). L'application peut également être en mesure de déclencher une interaction (par exemple un rendu de score de complémentarité électrostatique, suite au dépassement d'un seuil, ou une navigation suite à la détection d'un hot-spot intéressant).

- Le gestionnaire d'interaction interprète cette commande, puis il effectue une requête au superviseur, afin que celui-ci détermine les modalités les plus adéquates. Si le concepteur a prévu un rendu précis (qui suit par exemple les recommandations ergonomiques), ou si l'utilisateur en a demandé explicitement un lors de sa commande, ces propositions sont ajoutées à la requête.

- La requête est traduite et propagée par l'interpréteur vers le superviseur. Dans le cas où aucun rendu n'est proposé, le superviseur devra le déterminer complètement. Si des propositions accompagnent la requête, le superviseur devra les valider, les refuser ou les préciser. La décision du superviseur suit une démarche logique, à base de connaissances statiques, de connaissances dynamiques et de règles qui s'appliquent à ces connaissances. D'abord, les connaissances statiques renferment la sémantique du rendu multimodal. Elles sont composées des choix de distribution des informations sur les modalités. Ensuite, les connaissances dynamiques sont les éléments du contexte fournis par l'observateur. Enfin, un ensemble de règles logiques régit le comportement du superviseur : leur finalité est d'aboutir à une décision de rendu multimodal à partir des prédicats que forment les connaissances.

- Le résultat de la requête est traité par le gestionnaire d'interactions. Puis le rendu est généré par les moteurs de rendu appropriés, et transmis à l'utilisateur par l'intermédiaire des médias. Toutes les informations contextuelles sont mises à jour, tant au niveau de l'application que dans la base de contexte du superviseur. 


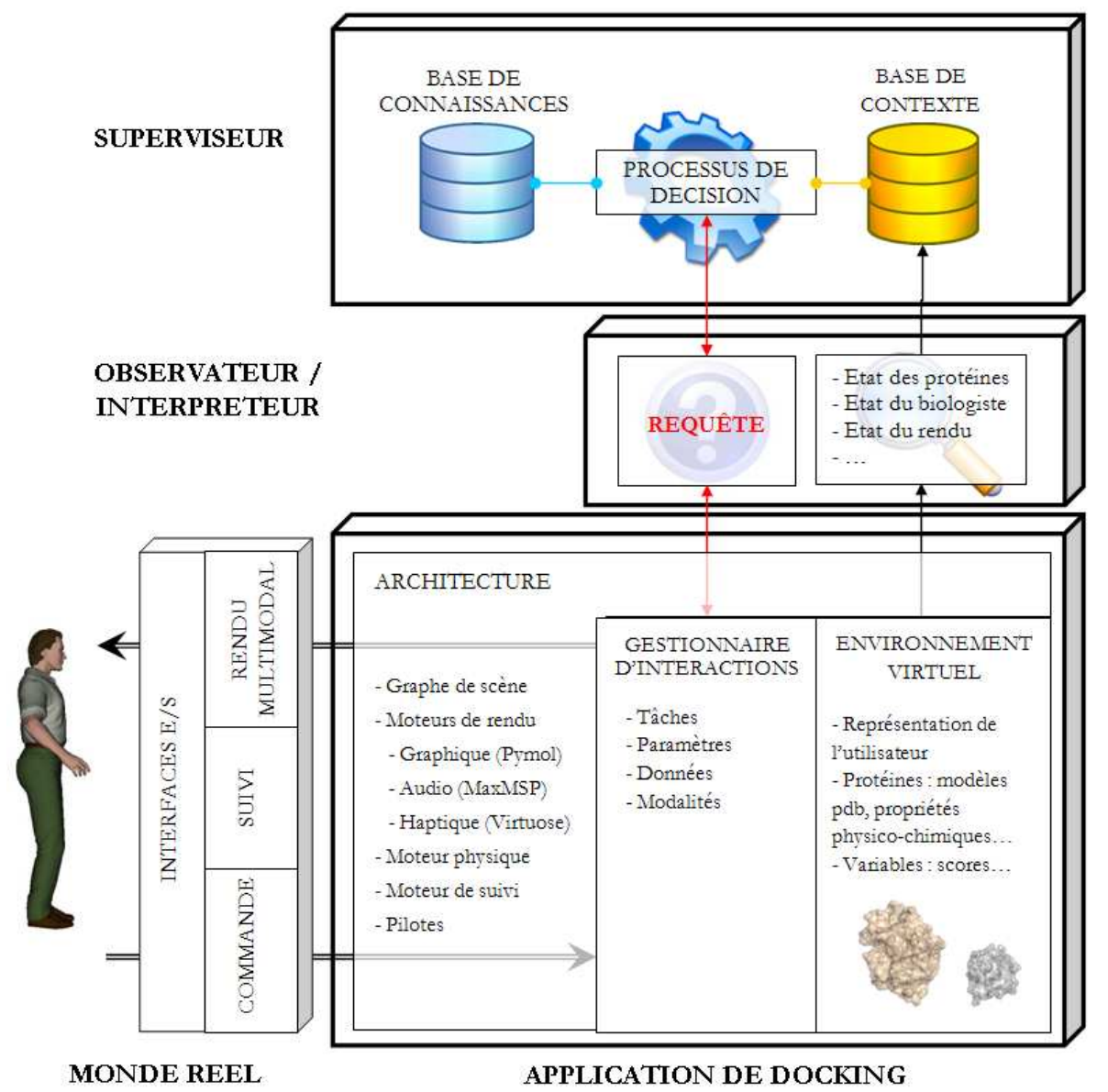

Figure 11. Architecture générale de la supervision multimodale.

\section{Evaluations en cours}

eval) Sur le plan de l'ergonomie, l'évaluation du prototype CoRSAIRe pour l'activité de docking, est réalisée suivant une approche itérative de conception-évaluation s'insérant dans la méthodologie de conception centrée-utilisateur plus globale du projet. Deux objectifs peuvent être schématiquement distingués dans le cadre des évaluations menées :

- évaluer l'environnement virtuel complet en termes d'utilité et d'utilisabilité pour la population de chercheurs concernés, la tâche de docking et le contexte dans lequel ils la réalisent; on notera qu'il s'agit moins ici de valider un système opérationnel que de valider la pertinence du concept ; 
- mettre au point et évaluer l'interface, notamment en ce qui concerne les techniques d'interaction et la représentation multimodale des informations.

Ces deux objectifs se traduisent par des méthodes et des situations différentes en termes de distance et de degré de complexité entre, d'une part, le dispositif et les sujets de la situation d'évaluation et, d'autre part, la situation d'usage avec les "véritables" utilisateurs dans un contexte professionnel réel. Evaluer le prototype sur son usage implique bien évidemment la mise en oeuvre de scénarios réalistes (i.e. impliquant des molécules et des données réelles accessibles selon de critères de représentativité) et la mobilisation d'utilisateurs également représentatifs. Inversement lorsqu'il s'agit d'établir les modalités de présentation de l'information et d'évaluer leur intelligibilité, il n'est pas systématiquement nécessaire d'avoir recours à des experts de la tâche.

Les évaluations menées jusqu'à présent l'ont été de façon formative, c'est-à-dire selon une approche consistant à évaluer tout au long du développement la qualité technique et ergonomique du prototype à un instant donné, en vue de fournir un retour à la conception et réorienter en conséquence les choix de conception. L'approche adoptée en fin de projet est dite sommative, c'est-à-dire qu'elle a pour objectif de mesurer la qualité et les propriétés en référence à des normes et des critères de performance externes. Ces études sont en cours.

\section{Conclusion}

Dans ce document, nous avons présenté un environnement immersif et multimodal pour aider le chercheur dans l'étude du phénomène de docking protéine-protéine. Cette approche a été conçue à partir des résultats d'une étude ergonomique des usages d'expert du domaine dans leur contexte de travail. Cette étude préliminaire avait pour principal objectif de prendre en compte les besoins des utilisateurs, de déterminer un ensemble de principes de conception, et d'intégrer la pratique des utilisateurs dans ce nouvel environnement. En proposant une approche dans laquelle l'utilisateur est impliqué en amont et en aval de procédures automatiques de docking, dans un environnement multimodal immersif, nous maximisons l'utilisation de son expertise. Nous rappelons que dans les pratiques classiques, des algorithmes automatiques fournissent un large ensemble de configurations de complexes à partir des données $3 \mathrm{~d}$ de deux protéines candidates. Les résultats de ces algorithmes restent longs à obtenir, car ils testent toutes les configurations géométriques possibles de docking de ces deux protéines. Ces configurations sont ensuite filtrées et regroupées par classes de manière semi-automatique selon des critères énergétiques et physico-chimiques. Enfin, le chercheur sélectionne au sein de cet ensemble quelques configurations à valider expérimentalement.

L'utilisation de l'expertise de l'opérateur en amont des méthodes automatiques dans un contexte immersif et multimodal permet à celui-ci d'exploiter en même temps ses capacités naturelles de détection de complémentarité de surface, ainsi que les connaissances bibliographiques ou implicites dont il dispose a priori par exemple sur 
la nature de l'interface entre les deux protéines, comme les hot-spots, etc. Ce processus permet de diminuer de façon importante le nombre de configurations que testeront les algorithmes automatiques utilisés ensuite. L'utilisation du contexte immersif et multimodal en aval de ces algorithmes automatiques est une aide au filtrage et à l'analyse des solutions proposées par les méthodes automatiques.

Le prototype de CoRSAIRe dédié au docking protéine/protéine est aujourd'hui achevé. Nous entrons désormais dans une phase d'évaluation, à la fois des rendus individuels, mais aussi de l'environnement complet, avec une attention particulière à l'apport du superviseur multimodal pour la gestion de la combinaison des rendus. Cette évaluation doit d'une part conclure sur la pertinence et l'efficacité de chaque rendu. Elle doit d'autre part se prononcer sur l'efficacité, en termes de durée et de qualité, de cette approche itérative dans la conception d'Environnements Virtuels Multimodaux, par rapport aux approches classiques.

Concernant les perspectives de ces travaux, nos principaux efforts vont porter sur l'intégration d'un modèle de protéines non rigide. En effet, dans de nombreux cas, les protéines ajustent leur structure afin de faciliter le processus de docking. La métaphore de type clé/serrure ou $L E G O$, utilisée jusqu'ici dans notre prototype et couramment employée dans les simulations de docking, est donc insuffisante pour étudier de façon virtuelle les phénomènes de docking. Cependant, les progrès des moteurs de dynamique moléculaire (GROMACS) ou l'utilisation d'approches nouvelles issues de la robotique (Lu et al., Aug 2005; Rossi et al., Jul 2007) qui considèrent les protéines comme des corps rigides articulés, permettent d'envisager à moyen terme une évolution de notre prototype. Dans ce contexte, pour atteindre les fréquences de rafraichissement nécessaires à certains canaux sensori-moteurs durant le rendu haptique, et nous étudions actuellement comment implémenter ces approches à l'aide de processeurs dédiés (GPU - Graphical Processor Unit, PPU - Physical Processor Unit) et de grilles de calcul (cluster de CPU).

\section{Remerciements}

Les travaux présentés dans ce papier ont été menés et se poursuivent avec le support de l'ANR (projet CoRSAIRe du programme ARA MDMSA) et avec le soutien du RTRA DIGITEO de la Région Ile-de-France (projet SIMCoD).

\section{Bibliographie}

Anastassova M., Mégard C., Burkhardt J., « Prototype evaluation and user-needs analysis in the early design of emerging technologies », 12th International Conference on HumanComputer Interaction, Beijing, China, 2007.

Anderson A., Weng Z., « VRDD : applying virtual reality visualization to protein docking and design », Journal of Molecular Graphics and Modelling, vol. 17, n 3, p. 180-186, 1999. 
André E., Handbook of natural language processing, chapter The generation of multimedia presentations, p. 305-327, 2000.

Andrusier N., al., « The Molecular Modeling Toolkit : A New Approach to Molecular Simulation. », J. Comp. Chem., vol. 21, p. 79-85, 2000.

Andrusier N., al., « FireDock : fast interaction refinement in molecular docking. », Proteins, vol. $69, \mathrm{n}^{\circ} 1, \mathrm{p} .139-59,2007$.

Annett J., Handbook of Cognitive Task Design, chapter Hierarchical Task Analysis, p. 17-35, 2003.

Baker N. A., Sept D., Joseph S., Holst M. J., McCammon J. A., « Electrostatics of nanosystems : Application to microtubules and the ribosome », Proceedings of the National Academy of Science, vol. 98, p. 10037-10041, August, 2001.

Barass S., Zehner B., « Responsive Sonification of Well-logs », International Conference on Auditory Display, Atlanta, Georgia, USA., 2000.

Berman H., Westbrook J., Feng Z., Gilliland G., Bhat T., Weissig H., Shindyalov I., Bourne P., « The Protein Data Bank», Nucleic Acids Res, vol. 1, n 28, p. 235-242, 2000.

Borrelli K., al., « PELE : Protein Energy Landscape Exploration. A Novel Monte Carlo Based Technique. », J. Chem. Theory Comput., vol. 6, n 1, p. 1304-1311, 2005.

Bouyer G., Rendu multimodal en Réalité Virtuelle : Supervision des interactions au service de la tâche., Ph.d. thesis, Université Paris XI, 2007.

Bouyer G., Bourdot P., Ammi M., « Supervision of 3D Multimodal Rendering for Proteinprotein Virtual Docking », Eurographics Symposium on Virtual Environments (EGVE), vol. 28, p. 49-56, 2008.

Comeau S., Gatchell W., Vajda S., Camacho C., « ClusPro : an automated docking and discrimination method for the prediction of protein complexes », Bioinformatics, vol. 20, $\mathrm{n}^{\circ} 1$, p. 45-50, 2004.

Connolly M., « Analytical molecular surface calculation », J. Appl. Crystallogr., vol. 16, p. 548558, 1983a.

Connolly M. L., « Solvent-accessible surfaces of proteins and nucleic acids », Science, vol. 221, p. 709-713, 1983b.

Corey R., Pauling L., « Molecular Models of Amino Acids, Peptides, and Proteins. », Review of Scientific Instruments, vol. 24, n 8, p. 621-627, 1953.

Cruz-Neira C., Sandin D., DeFanti T. A., Kenyon R. V., Hart J., « The CAVE : Audio Visual Experience Automatic Virtual Environment », SIGGRAPH, vol. 35, p. 64-72, 1992.

Ferey N., Delande O., Grasseau G., Baaden M., « A VR Framework for Interacting with Molecular Simulations », ACM-VRST 2008 : In proceedings of Virtual Reality Sofware and Technologies, in press, 2008.

Fernandez-Recio J., Totrov M., Abagyan R., « ICM-DISCO docking by global energy optimization with fully flexible Side-Chains », Cambridge, MA : Bradford Books / MIT Press, vol. $1, \mathrm{n}^{\circ}$ 52, p. 113-117, 2003.

Frederick P. Brooks J., Ouh-Young M., Batter J. J., Kilpatrick P. J., « Project GROPE : Haptic displays for scientific visualization », SIGGRAPH '90: Proceedings of the 17th annual conference on Computer graphics and interactive techniques, ACM, New York, NY, USA, p. $177-185,1990$ 
Garcia-Ruiz M., Guttierez-Pulido J., « An overview of auditory display to assist comprehension of molecular information », Interacting with Computers, vol. 18, n 4, p. 853-868, 2006.

Ghiglione R., Landré A., Bromberg M., Molette P., L’analyse automatique des contenus, 1998.

Gottschalk S., Lin M., Manocha D., « OBBTree : A Hierarchical Structure for Rapid Interference Detection », Computer Graphics, vol. 30, p. 171-180, 1996.

Grosdidier A., Conception d'un logiciel de docking et applications dans la recherche de nouvelles molécules actives, PhD thesis, Université Joseph Fourier Grenoble 1, 2007.

Hart T., Read R., « A multiple-start Monte Carlo docking method», Proteins : Structure, Function, and Genetics, vol. 13, n 3, p. 206-222, 2004.

Hess B., al., « GROMACS 4 : Algorithms for Highly Efficient, Load-Balanced, and Scalable Molecular Simulation. », J. Chem. Theory Comput., vol. 4, n 3, p. 435-447, 2008.

Kitagawa M., Dokko D. ., Okamura A. ., Yuh D. ., « Effect of sensory substitution on suturemanipulation forces for robotic surgical systems », Journal of Thoracic and Cardiovascular Surgery, vol. 129(1), p. 151-158, 2005.

Levine D., Facello M., Hallstrom P., Reeder G., Walenz B., Stevens F., « Stalk : an interactive system for virtual molecular docking », In Proceedings of IEEE Computational Science and Engineering, vol. 4, $\mathrm{n}^{\circ}$ 2, p. 55-65, 1997.

Lu T.-C., Ding J., Crivelli S., « DockingShop : a tool for interactive protein docking », Computational Systems Bioinformatics Conference, 2005. Workshops and Poster Abstracts. IEEE, vol. , p. 271-272, Aug 2005.

Lundin K. E., Sillen M., Cooper M. D., Ynnerman A., « Haptic visualization of computational fluid dynamics data using reactive forces », in R. F. Erbacher, J. C. Roberts, M. T. Gröhn, K. Börner (eds), Visualization and Data Analysis 2005. Proceedings of the SPIE, Volume 5669, pp. $31-41$ (2005)., vol. 5669 of Presented at the Society of Photo-Optical Instrumentation Engineers (SPIE) Conference, p. 31-41, March, 2005.

Maciejewski R. andChoi S., Ebert D., Tan H., « Multi-Modal Perceptualization of Volumetric Data and Its Application to Molecular Docking », First Joint Eurohaptics Conference and Symposium on Haptic interfaces For Virtual Environment and Teleoperator Systems, 2005.

Pipe S., « Recombinant clotting factors », Thromb Haemost, vol. 99, n 5, p. 840-50, May 2008.

Ray N., Cavin X.and Paul J., Maigret B., « Intersurf : dynamic interface between proteins », Journal of Molecular Graphics and Modelling, vol. 23, n 4, p. 347-354, 2005.

Richard P., Chamaret D., Inglese F.-X., Lucidarme P., Ferrier J.-L., « Human-Scale Haptic Virtual Environment for Product Design : Effect of Sensory Substitution », The International Journal of Virtual Reality, vol. 5(2), p. 37-44, 2006.

Ritchie D., « Evaluation of protein docking predictions using Hex 3.1 in CAPRI rounds 1 and $2 »$, Proteins, vol. 52, n 1, p. 98-106, 2003.

Rossi R., Isorce M., Morin S., Flocard J., Arumugam K., Crouzy S., Vivaudou M., Redon S., « Adaptive torsion-angle quasi-statics : a general simulation method with applications to protein structure analysis and design. », Bioinformatics, vol. 23, n 13, p. i408-17, Jul 2007.

Sanner M., Olson A., Spehner J.-C., « Reduced Surface : An Efficient Way to Compute Molecular Surfaces », Biopolymers, vol. 38, p. 305-320, 1996.

Seeger A., Chen J., « Controlling Force Feedback Over a Network », The Second PHANToM User's Group Workshop, Cambridge, Massachusett, USA, MIT, 1997. 
Touraine D., Bourdot P., Bellik Y., Bolot L., « A Framework to Manage Multimodal Fusion of Events for Advanced Interactions within Virtual Environments », International EUROGRAPHICS Workshop on Virtual Environment, (EGVE 2002), Barcelona, Spain, 2002.

Villoutreix B., Bastard K., Sperandio O., Fahraeus R., Poyet J., Calvo F., Deprez B., Miteva M., «In silico-in vitro screening of protein-protein interactions : towards the next generation of therapeutics », Curr Pharm Biotechnol, vol. 9, n² 2, p. 103-22, Apr 2008.

Wang R., Lu Y., Wang S., « Comparative Evaluation of 11 Scoring Functions for Molecular Docking », Journal of medicinal chemistry, vol. 46, p. 2287-2303, 2003.

Wriggers W., Birmanns S., «Interactive fitting augmented by force-feedback and virtual reality », J. cell biol., vol. 144, p. 123-131, 2003.

Zacharias M., « ATTRACT : protein-protein docking in CAPRI using a reduced protein model.», Proteins, vol. 60, n 2, p. 252-6, 2005. 